\title{
FAST-WAVE HEATING OF A TWO-COMPONENT PLASMA
}

BY

THOMAS $\mathrm{H}$, STIX

\section{PLASMA PHYSICS LABORATORY}

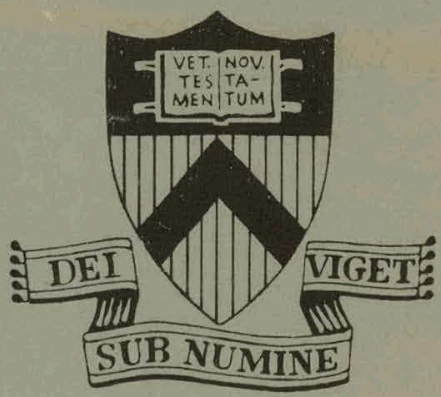

\section{PRINCETON




\section{DISCLAIMER}

This report was prepared as an account of work sponsored by an agency of the United States Government. Neither the United States Government nor any agency Thereof, nor any of their employees, makes any warranty, express or implied, or assumes any legal liability or responsibility for the accuracy, completeness, or usefulness of any information, apparatus, product, or process disclosed, or represents that its use would not infringe privately owned rights. Reference herein to any specific commercial product, process, or service by trade name, trademark, manufacturer, or otherwise does not necessarily constitute or imply its endorsement, recommendation, or favoring by the United States Government or any agency thereof. The views and opinions of authors expressed herein do not necessarily state or reflect those of the United States Government or any agency thereof. 


\section{DISCLAIMER}

Portions of this document may be illegible in electronic image products. Images are produced from the best available original document. 
Fast-Wave Heating of a Two-Component Plasma*

\author{
THOMAS H. STIX \\ Plasma Physics Laboratory, Princeton University \\ Princeton, New Jersey 08540 USA
}

\title{
ABSTRACT
}

The use of the compressional hydromagnetic mode (also called the magnetosonic or, simply, the fast wave) is examined in some detail with respect to the heating of a tritium plasma containing a few percent deuterium. Efficient absorption of wave energy by the deuteron component is found when $\omega=\omega_{c}$ (deuterons), with $\therefore \quad Q_{\text {wave }} \geqslant 100$. Reasonable efficiencies are found also for electron heating, but coherence effects between transittime and Landau damping for electrons reduce the total absorption for both processes to one-half of the transittime power, calculated separately.

The fusion output of a two-component neutralinjected plasma can be enhanced by selective heating of the injcctcd deuterons. Also, selective deuteron absorption may be used for ion-tail creation by radiofrequency excitation alone, as an alternative to neutral injection. The dominant behavior of the high-energy deuteron distribution function is found to be $f(v) \sim \exp \left[(3 / 2) \int^{v} \mathrm{dv}\langle\Delta v\rangle /\left\langle\left(\Delta v_{\perp}\right)^{2}\right\rangle\right]$, where $\langle\Delta v\rangle$ is the Chandrasekhar-spitzer drag coefficient, and $\left\langle\left(\Delta v_{\perp}\right)^{2}\right\rangle$ is the Kennel-Englemann quasilinear diffusion coefficient 
for wave-particle interaction at the deuteron cyclotron frequency. An analytic solution to the one-dimensional Fokker-Planck equation, with rf-induced diffusion, is developed, and using this solution together with Duane's fit to the $D-T$ fusion cross-section, it is found that the nuclear fusion power output from an rf-produced twocomponent plasma can significantly exceed the incremental (radiofrequency) power input. 


\section{INTRODUCTION}

The need for supplementary heating for a tokamak - supplementary, that is, to the ohmic heating associated with the toroidal current - has been recognized for a number of years. Only recently, however, have the special benefits become clear for a specific form of supplementary heating: putting the heat in as a high-energy tail on the ion distribution. In bringing a classical (50-50) DT reactor to ignition, the high energy ions cause fusion reactions which release alpha particles and enhance the heating power, while in the two-component fusion device [1], the creation of the ion tail is an essential element of the total concept. The most direct way to produce the ion tail is evidently by neutral injection. It will be some years, however, before neutral beam technology allows us to test this heating method at the requisite beam currents and voltages, and the final answers on efficiency, penetration, impurities, plasma stability, and beam slowdown rates must await such testing. Meanwhile, it is appropriate to look at radiofrequency methods as an alternative process for plasma heating and for ion tail creation. Radiofrequency heating can play a number of roles:

a. Electron heating. A successful two-component fusion experiment needs an electron temperature of $5 \mathrm{keV}$ or more. Neutral injection provides supplementary heat to both the background ions and electrons, and too much background ion heating can be wasteful. In addition, it may turn out that rf electron heating is less costly to install than injection heating with similar capability. 
b. Supplementary heating. 'Supplementary ion heating is the traditional role assigned to rf heating. c. Ion tail creation. Selective absorption of rf energy by a.minority of the plasma ions may be achieved by cyclotron resonance tuned to the minority ions [2-4], or by cyclotron harmonic heating which preferentially delivers power to the high energy (large Larmor radius) component [4-61: d. Ion tail enhancement. Radiofrequency heating may be used to enhance neutral injection itself by tuning the rf to resonance with the injected beam particles in the plasma. Such heating can easily add perpendicular energy to beam ions, and can increase and maintain the energy dispersion of the beam and the fusion" reaction rate. The wave mode which is uncannily well suited to the various objectives outlined above is the compressional hydromagnetic wave, also called the magnetosonic mode or simply the fast wave: The wave lengths in the desired frequency $\left(\omega \sim \omega_{\mathrm{Ci}}\right)$ and density $\left(\mathrm{n} \sim 10^{14} \mathrm{~cm}^{-3}\right)$ ranges are long enough to allow reasonable mode separation in the large tokamak plasmas and good coupling to the antenna structures. Fast wave heating [7] for large plasma devices was first seriously examined by Adam and Samain[4] . On the experimental side, experiments in 1967 on the Model C-stellarator showed both fast-wave generation and plasma heating [8]. "More recently, the 1974 ST tokamak experiments [9] strongly confirmed fast wave heating and, equaljy important, demonstrated the existence of and showed the ability to excite high-Q discrete fast-wave toroidal modes [5]. By proper selection of the fast-wave frequency, mode numbers, 
magnetic field strength and plasma composition, it is possible to heat ions and/or electrons, to vary the deposition pattern of the heating, and to modify the ion velocity distribution. Using fastwave heating equipment, the experimentalist therefore has available to him, with rather modest parameter changes, a great flexibility in his experimental approach and a great range of physical processes.

In this paper we examine in some detail the specific use of fast-wave excitation for heating large magnetically-confined toroidal plasmas. We look at electron heating through waveabsorption via Landau and transit-time damping (the two effects are coherent and cross-terms must be considered), and ion heating through cyclotron damping. A Fokker-Planck calculation with a quasilinear-type rf diffusion coefficient shows that selective rf cyclotron absorption is competitive with neutral injection for the formation of a fusion-producing two-component ion velocity distribution.

\section{ALLOWED MODES AND MODE-TRACKING}

We start our detailed consideration of fast-wave heating by looking at the properties of this mode in a homogeneous cold plasma immersed in a uniform static magnetic field. The wave equation can be written in the usual matrix form, with $k_{y}$ set equal to zero,

$$
\left(\begin{array}{lll}
k_{x x}-n_{z}^{2} & k_{x y} & n_{x z} \\
k_{y x} & k_{y y}-n^{2} & 0 \\
n_{z} n_{x} & 0 & k_{z z}-n_{x}^{2}
\end{array}\right),\left(\begin{array}{c}
E_{x} \\
E_{y} \\
E_{z}
\end{array}\right)=0
$$


$\mathrm{n}_{\mathrm{x}} \equiv \mathrm{k}_{\mathrm{x}} \mathrm{c} / \mathrm{\omega}$ and $\mathrm{n}_{\mathrm{z}} \equiv \mathrm{k}_{\mathrm{z}} \mathrm{c} / \mathrm{w}$ : are the two remaining components of the vector refractive index, $n^{2}=n_{x}^{2}+n_{z}^{2}$, and we have neglected components of the dielectric tensor, $\vec{K}$, which are finite only for $T \neq 0$. The usual low-frequency high-conductivity approximation is now taken, $\left|\mathrm{K}_{z z}\right|>>\mathrm{n}_{\mathrm{x}^{\prime}}^{2} \mathrm{n}_{\mathrm{xz}}{ }^{\prime}$ and we may further denote $\mathrm{K}_{\mathrm{xx}} \simeq \mathrm{K}_{\mathrm{yy}}=\mathrm{s}=(1 / 2)(\mathrm{R}+\mathrm{L})$ and $\mathrm{K}_{\mathrm{xy}}=-\mathrm{K}_{\mathrm{yx}}=-i \mathrm{D}:=-(i / 2)(\mathrm{R}-\mathrm{L})$, leaving

$$
\frac{1}{4}\left(\begin{array}{cc}
R+L-2 n_{z}^{2} & -i(R-L) \\
\cdots & \\
i(R-L) & R+L-2 n^{2}
\end{array}\right)\left(\begin{array}{c}
E_{x} \\
E_{y}
\end{array}\right) \simeq 0
$$

For $|\omega| \ll \omega_{c e} \omega_{\text {pe }}$ and neglecting displacement current, but including the electron contribution, the cold-plasma dielectric tensor elements are $R \simeq\left(4 \pi n_{i} m_{i} c^{2} / B^{2}\right)\left[\omega_{c i} /\left(\omega_{c i}+\omega\right)\right]$;

$L \simeq\left(4 \pi n_{i} m_{i} c^{2} / B^{2}\right)\left[\omega_{c i} /\left(\omega_{c i}-\omega\right)\right]$. The dispersion relation for hydromagnetic waves is given just by the determinant of the $2 \times 2$ matrix, and may then be written

$$
N_{\perp}^{2}=A-N_{11}^{2}+\frac{A(I-A)}{A-N_{n}^{2}}
$$

where $\mathrm{N}_{\mathcal{L}}$ and $\mathrm{N}_{\|}$, the Alfvén refractive indices, are defined

$$
\begin{aligned}
& \mathrm{N}_{\perp}^{2} \equiv \mathrm{n}_{\mathrm{x}}^{2}\left(\frac{\mathrm{B}^{2}}{4 \pi \mathrm{n}_{i} \mathrm{~m}_{i} \mathrm{c}^{2}}\right) \\
& \mathrm{N}_{n}^{2} \equiv \mathrm{n}_{\mathrm{z}}^{2}\left(\frac{\dot{B}^{2}}{4 \pi \mathrm{n}_{i} \mathrm{~m}_{i} \mathrm{c}^{2}}\right) \\
& \mathrm{A} \equiv \omega_{\mathrm{Ci}}^{2} /\left(\omega_{\mathrm{C} i}^{2}-\omega^{2}\right)
\end{aligned}
$$


The dispersion relation is sketched in Fig. 1 for the two cases, $A>I\left(\omega<\omega_{C i}\right)$ and $A<0\left(\omega>\omega_{C i}\right)$. The curve for $\omega>\omega_{C i}$ shows the two well-known Alfvén waves, the shorter-wavelength shear or ion cyclotron mode, and the longer-wavelength compressional mode, also called the magnetosonic or fast wave. The case of especial interest to us is that where the plasma ions are predominatly tritons, but where $\omega$ is set equal to the deuteron cyclotron treqeuncy. In this case $A--4 / \mathrm{s}$. It 13 instruotive to draw the dispersion relation for just the propagating region of the fast wave, using $\mathrm{N}_{\mathcal{1}}, \mathrm{N}_{1}$ as coordinates rather than $\mathrm{N}_{\perp}^{2}$ and $\mathrm{N}_{\|}^{2}$, and such a plot is shown in Fig. 2. Now in a finite-geometry plasma, wavenumbers for the allowed modes will be approximate multipjes of some fundamental wavenumbers, e.g., $\mathrm{N}_{11} \simeq n \mathrm{~N}_{n}^{(0)}, \mathrm{N}_{\perp} \simeq \mathrm{N}_{\perp}^{(0)}$. Therefore, the natural modes of the finite-geometry plasma will occur where the dispersion relation curve passes through an intersection of the background cartesian grid laid out with spacings corresponding to the fundamental wavenumbers, as indicated in Fig. 2. As the device size and plasma density are increased, the grid spacings in Fig. 2 will decrease. For instance, in a torus [10],

$$
\begin{aligned}
& N_{n}^{(0)}=\frac{c}{R \omega}\left(\frac{B^{2}}{4 \pi n_{i} m_{i} c^{2}}\right)^{1 / 2}, \\
& N_{\perp}^{(0)} \simeq \frac{\pi c}{a \omega}\left(\frac{B}{4 \pi n_{i} m_{i} c^{2}}\right)^{1 / 2} .
\end{aligned}
$$


Actually the mode spectrum is sightly more complicated than just described. The approximation that $N_{\perp} \simeq v N_{\perp}^{(0)}$. with $N_{\perp}^{(0)}$ given by Eq. 4 is valid for modes with toroidal and radial but no poloidal variation. But the complete dispersion relation, for a toroidal system will have a structure similar to that given by Bernstein and Trehan [11] for a finite-radius cylinder of uniformdensity cold plasma: the field amplitude varies as $B_{2}^{(1)}=$ constant $J_{m}(v r) \exp (i m \theta+i k z-i \omega t)$ and the dispersion relation is of the form

$$
\frac{k_{\perp} a J_{m}^{\prime}\left(k_{\perp} a\right)}{J_{m}\left(k_{\perp} a\right)}=A+m B
$$

where $A$ and $B$ are functions of $\omega, k_{n}$, and the plasma parameters. Solutions of this dispersion relation appear between each pair of zeros of $J_{m}\left(k_{\perp} a\right)$ and therefore can be categorized simply by $k_{\perp} a \simeq \pi(r+|m / 2|)+\varepsilon(r, m)$, where $r$ and $m$ are the radial and poloidal mode numbers, and $\varepsilon$ is an angle of magnitude less than $\pi$. The integer quantity we had called $v$ would be $\sim k_{\perp} a / \pi$, but we see now that the complete spectrum shows modes at half-integral values of $\nu$, for $\nu>1$, and each such mode has a $(2 v-1)$-fold multiplicity, i:e., $v=1$ corresponds to $\mathrm{r}=1, \mathrm{~m}=0 ; \quad \nu=3 / 2$ to $\mathrm{r}=1, \mathrm{~m}= \pm 1 ; \nu=2$ to $\mathrm{r}=2, \mathrm{~m}=0$ and $\mathrm{r}=1, \mathrm{~m}= \pm 2$, etc:

The mode spectrum for a straight cylinder is degenerate with respect to propagation parallel or antiparallel to $\hat{\mathbf{z}}_{\mathrm{B}}$, i.e., with respect to $\pm k_{1}$. It has been pointed out by chance and Perkins [12] that this degeneracy is resolved in a tokamak plasma, the line separation being related to mode rotation due to the 
helical magnetic lines of force. Just as each designated v-value in Fig. 2 is actually a $(2 v-1)$-fold multiplet (not shown on the drawing), it is then also true that each $n$ value actually designates a doublet (not shown) in the spectrum. Certain important qualitative conclusions can be drawn immediately from this interpretation of Fig. 2. We consider the possibilities for fixed $\omega / B$, corresponding to setting $\omega=\omega_{c j}$ for a $j$-type i.on 1

1. For a toroidal device which is too sinali in minor radus, or in which the desired density has too small a value, even the nearest grid intersection will fall outside the dispersion curve, and no natural fast-wave modes will propagate. The Princeton c-stellarator plasma fell in this category.

2. For an intermediate-size plasma, a small number of modes will be possible, and these will be reasonably well separated in density. The recent fast-wave experiments in ST fall into this category

3. For a somewhat larger plasma, many modes will be possible, but the mode separation in density will still be large enough that rather broad-spectrum excitation can be used successfully to excite the desired single modes. Princeton's PLT, PDX, and perhaps TFT'R would be in this category.

4. For reactor-size plasmas, the dense mode spectrum will tend to frustrate efforts to excite optimum single modes to achieve, say, ion heating without electron Landau damping, or core heating "without surface heating. In such large devices, then, considerable attention will have to be paid to the use of antenna structures which narrow the $k_{\perp}$ and $k_{\|}$excitation spectra. 
5. For any plasma, the occurrence of a natural mode with specified $k_{1}, k_{1}, w$, and $B$ requires the proper value for ion density. In pulsed plasmas and in the start-up of steadystate plasmas, the density will vary with time, and allowance must be made for tracking resonant conditions either by varying $\omega$ or, jumping modes, by changing the $\vec{k}$-excitation spectrum.

\section{3. $\vec{E}-F I E L D ~ P O L A R I Z A T I O N$}

The natural sense of rotation of ions around the magnetic field lines is that of left-handed screw motion progressing along the $\vec{B}$ vector. Cyclotron acceleration of the ions therefore depends principally on the amplitude of the left-handed circularlypolarized component of the wave electric field in the plasma.. In detail; if one goes to finite-Larmor-radius theory, it will be found that ion acceleration due to a pure right-hand E-field component will be weaker by a factor $\quad \sim \mathrm{k}_{\perp}^{4}<\rho{ }_{\mathrm{Li}}^{4}>\quad$ than that due to the left-hand component, a result which holds even for cyclotron-harmonic acceleration. (Examine the structure of $\left|\theta_{n, k}\right|^{2}$ in Eq. (18) below.) Going back to Eq. (2), we can determine the ratio of the left-hand $\vec{E}$ component $\left(E_{x}+i E_{Y}\right)$ to the righthand component $\left(E_{x}-i E_{y}\right)$,

$$
\frac{E_{x}+i E_{y}}{E_{x}-i E_{y}}=-\frac{R-n_{z}^{2}}{L-n_{z}^{2}}=\frac{\frac{\omega_{C i}}{\omega+\omega_{C i}}-N_{" \prime}^{2}}{\frac{\omega_{C i}}{\omega-\omega_{C i}}+N_{\prime \prime}^{2}}
$$

and provided that $\omega>\omega_{\mathrm{Ci}}$ and $\omega_{\mathrm{Ci}} /\left(\omega+\omega_{\mathrm{Ci}}\right)>\mathrm{N}_{\|}^{2}>0$ (that is, for a propagating fast wave at $\left.\omega>\omega_{c i}\right)$, then 


$$
\frac{E_{x}+i E_{y}}{E_{x}-i E_{y}}<\frac{\omega-\omega_{C i}}{\omega+\omega_{C i}}
$$

For $\omega=(3 / 2) \omega_{C i}$, corresponding to deuteron cyclotron resonance in a triton plasma, the right-hand side of (6) equals $1 / 5$. The ratio in (5) is a maximum for $\mathrm{N}_{\|}^{2}=0$ and drops to zero for parallel propagation, which is characterized by the dispersion relation $n_{n}^{2}=R \quad\left(n_{\perp}^{2}=0\right)$ and pure right-hand circular polarization. ('the $E_{L} / E_{R}$ relation, Eq. (5), applies, of course, also to ion cyclotron waves, but reduction of $E_{L}$ is avoided in this instance because $n_{z}^{2} \sim L$. For dense plasmas, this condition on $n_{z}^{2}$ requires very short parallel wave-lengths.) The relative magnitude of the left-hand $E$. field is also reduced if the number of resonant ions is increased - up to now, we have neglected the contribution of the resonant deuterons to the dielectric tensor. 'l'helr principal cuntribution is to $L$, but hot-plasma theory limits the magnitude even at exact resonance. Writing $L=L_{T}+L_{D}$ for the tritium (plus electrons) and deuterium. components, one will find the extremum value for $L_{D} / L_{T}$, occurring at $\omega=\omega_{\mathrm{CD}}$

$$
\left(\frac{L_{D}}{\bar{L}_{T}}\right)^{\text {extr. }}=-\frac{1 \pi}{3}^{1 / 2} \frac{n_{\nu}}{n_{T}} \frac{(1)}{C \nu}^{\mid k_{z}}\left(\frac{m_{D}}{2 k T_{D}^{(I I)}}\right)^{1 / 2} .
$$

The power going into deuteron heating will be proportional to $n_{D}\left|E_{x}+i E_{Y}\right|^{2}$ and will be a maximum for $n_{D}$ such that $\left|L_{D} / L_{T}\right|^{\text {extr. }}$ is of the order of unity. Typically this leads to optimal fractional deuteron concentrations of a few percent. A similar argument 
can be applied to second-harmonic heating in, for instance, a 50-50 D-T plasma. In that case, however, the contribution to $\mathrm{L}$ from the resonant ions is reduced by a factor proportional to $k_{\perp}^{2}<\rho \frac{2}{L i}>$

\section{ION CYCLOTRON DAMPING}

Collisionless cyclotron damping can take place with absorption of wave energy by the particles that, in their own frame of reference, feel the wave E-field at their cyclotron frequency. The well known condition for such single-particle resonance is $\omega-k_{n} v_{n}=\omega_{c}$. We will return to the standard uniform-B calculation, but it is instructive first to calculate particle heating in the inhomogeneous tokamak magnetic field. Given $\omega, k_{n}$, and

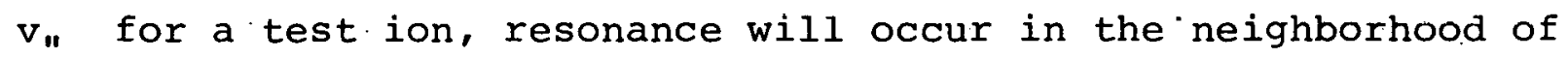
a certain $B=$ constant surface. Ions following along the B-lines which twist around the magnetic axis can pass through the resonant surface twice each complete revolution. On each passage through resonance the ions receive a kick in $\mathrm{mv}_{\perp}$ which is not difficult to calculate. The single-particle equations of motion perpendicular to $\vec{B}$ are

$$
\begin{aligned}
& \dot{\mathrm{v}}_{\mathrm{x}}-\Omega(t) \mathrm{v}_{\mathrm{y}}=\frac{\mathrm{q}}{\mathrm{m}} \mathrm{E}_{\mathrm{x}} \cos \omega t \\
& \dot{\mathrm{v}}_{\mathrm{y}}+\Omega(t) \mathrm{v}_{\mathrm{x}}=-\frac{\mathrm{q}}{\mathrm{m}} \mathrm{E}_{\mathrm{y}} \sin \omega t
\end{aligned}
$$

Let $E_{ \pm} \equiv(l / 2)\left(E_{x} \pm E_{y}\right), u \equiv v_{x}+i v_{y} \cdot \Omega_{l}(t)$ is the cyclotron frequency at the instantaneous particle position. Expand $\Omega(t)$ around the resonant surface, 


$$
\Omega(t)=\omega+\left(t-t_{0}\right) \Omega^{\prime}(t)+\ldots
$$

The two real-variable equations can then be written as a single equation in the complex variable $u$,

$$
\frac{d u}{d t}+i \Omega(t) u=\frac{g}{m}\left(E_{+} e^{-i \omega t}+E_{-} e^{i \omega t}\right)
$$

We neglect the non-resonant ( $\left.E_{-}\right)$driving term and integrate,

$$
\begin{aligned}
u(t) \exp \left(i \int_{-\infty}^{t} \Omega d t\right) & =u(-\infty)+\frac{q}{m} E_{+} \int_{-\infty}^{t} d t^{\prime} \exp \left[\frac{i \Omega^{\prime}\left(t^{\prime}-t_{0}\right)^{2}}{2}\right] \\
& +u(-\infty)+\frac{q}{m} E_{+}\left(\frac{2 \pi i}{\Omega^{\prime}}\right)^{1 / 2}
\end{aligned}
$$

for $t \gg t_{0}$. We assume that $u(-\infty)$ is randomly phased with respect to $E_{+}$(but will return to this point in Section 8 ) and compute the average change in energy per transit of the resonant surface,

$$
\frac{m}{2}<u(t) u(t) *-u(-\infty) u(-\infty) *>=\frac{m}{2}\left|\frac{g}{m} E_{+}\right|^{2} \cdot\left|\frac{2 \pi}{\Omega^{\prime}}\right| \equiv w(\vec{r}) .
$$

To find the total rate of power absorption per unit volume, P, we multiply the energy gained per transit, $W$ by the total ion transiting rate, $v$, forming $P=v(\vec{r}) W(\vec{r})$. For this calculation we choose a local coordinate system in which $\hat{z}$ is the direction of the toroidal component of $\vec{B}, \hat{x}$ is in the direction of the major radius, and $\hat{y}$ is vertical, parallel to the major axis of the torus. The exact location of the resonant surface for each ion depends on 
$v_{n}$ in order to satisfy $\omega-k_{n} v_{\|}-\omega_{C}=0$, and $v(x, y)$ is the number of ion transits across such resonant surfaces that occur per unit time in each unit volume of the plasma,

$$
|v d x|=\int_{u}^{u+\Delta u} d v_{z} \int_{-\infty}^{\infty} d v_{x} \int_{-\infty}^{\infty} d v_{y}\left|v_{x g}\right| f(x, y, \vec{v}),
$$

where

$$
u=v_{n}^{(\text {res })} \equiv \frac{\omega-\omega_{c}(x)}{k_{n}}
$$

and

$$
u+\Delta u=\frac{\omega-\omega_{E}(x+\Delta x)}{k_{n}},
$$

and where $v_{x g}$ is the $x$-component of the ion guiding-center velocity. From these equations we derive

$$
\Delta u=\Delta x \frac{d v_{n}^{(\text {res })}}{d x}=-\Delta x\left(\frac{1}{k_{n}} \frac{d}{d x} \omega_{c i}\right)
$$

Also, we may write

$$
\Omega^{\prime}=\frac{d \Omega}{d t}=\frac{d}{d t} \omega_{c i}=v_{x g} \frac{d}{d x} \omega_{c i}
$$

where $v_{x g}$ is again the $\hat{x}$ guiding-center velocity. In forming $\mathrm{P}$, it is interesting to note that $\mathrm{v}_{\mathrm{xg}}$ cancels out, leading to a result which is independent of the local value of tokamak rotational transform, etc. We find simply

$$
P(\vec{r})=\frac{\pi q^{2}}{m\left|k_{11}\right|} \int d v_{x} d v_{y} f\left(\vec{r}, \vec{v}_{\perp}, v_{n}^{(\text {res })}\right)\left|E_{+}(\vec{r})\right|^{2}
$$

which is precisely the result one obtains [13] from conventional uniform-B hot-plasma theory. This identity of results has the 
further welcome consequence that the use of the uniform-B-theory monochromatic-spectrum quasilinear diffusion coefficient may now be justified for the calculation of ion heating in the inhomogeneous B-field of a tokamak. The point will be discussed in Section 7 . The next step is to average $P(\vec{r})$ over the volume between two magnetic surfaces, of minor radii $r_{1}$ and $r_{2}$. For this purpose we write

$$
f\left(\vec{r}, v_{n}(\text { res })\right)=E\left[\vec{r}, \frac{\omega-\omega_{c i}}{k_{n}}\right] \quad,
$$

and

$$
d x=d \omega_{c i} /\left(d \omega_{c i} / d x\right)=-\frac{R}{\omega_{C i}} d \omega_{c i}
$$

and then

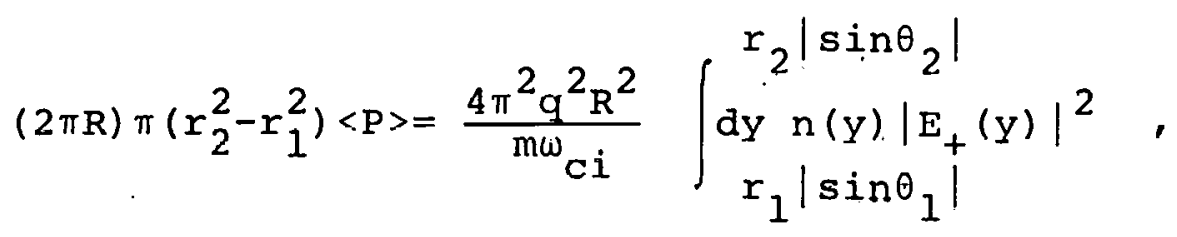

where $n(y), m$ and $\omega_{C i}$ refer to the resonant ions only (e.g., deuterons) and where the integral is carried out along $y$ at a value of major radius (x) corresponding to the center of the resonant region. See Fig. 3. Symmetry for $\pm Y$ is assumed.

$\theta_{1}$ and $\theta_{2}$ are the angles between the minor radii, $r_{1}$ and $r_{2}$ ' and the $x$-axis. The heat which is first generated in the two resonant regions then is quickly distributed, by the flow of ions along the magnetic lines of force, to the volume between the torus-shaped magnetic surfaces with minor radii $r_{1}$ and $r_{2}$. 
For $r_{2}$ and $r_{1}$ close together, the average power per unit volume to the resonant ions, of density $n(r)$, on a toroidal magnetic surface with minor radius $r$ is simply

$$
\langle\mathrm{P}\rangle=\frac{\mathrm{n}(\mathrm{r}) \mathrm{Zec}}{\mathrm{B}} \frac{\mathrm{R}}{\mathrm{r}|\sin \theta|}\left|\mathrm{E}_{+}\right|^{2} \text { : }
$$

This same result, averaged over the tokamak cross-section, was already obtained by Adam and Samain [14].

A happy consequence of this rf heating is the appearance of a geometrical effect which favors the deposition of heat in the core (small $r$ ) of the tokamak plasma.

\section{ELECTRON LANDAU AND TRANSIT-TIME DAMPING}

Landau damping and transit-time damping both involve energy absorption by particles moving along $\vec{B}$ with the phase velocity of the wave, i.e., particles for which $\omega-k_{n} v_{\|}=0$. In Landau damping, the force on the particle due to the wave is $q \vec{E}$; for transit-time damping the acting force is $-\mu \nabla B$. Both processes are effective in fast-wave damping by the thermal electrons. The exact calculation is tedious in its algebra as the two effects are coherent and cross-terms need be retained. Symbolically, the power abosrbed per unit volume is given simply by

$$
P=-\frac{i \omega}{16 \pi} \vec{E}^{\star} \cdot \overrightarrow{\mathrm{K}} \cdot \overrightarrow{\mathrm{E}}+\text { complex conjugate, }
$$

in which $\vec{E}$ is the complex amplitude in the representation of the real wave field $\vec{E}_{O^{\prime}} \vec{E}_{0}=\operatorname{Re}\{\vec{E} \exp (-i \omega t+i \vec{k} \cdot \vec{r})\}$, and $\vec{K}$ is the 
usual hot plasma dielectric tensor. The only terms of $\vec{k}$ that need be retained in the expression for $P$ are the collisionless absorption terms from the $\omega-k_{n} v_{n}=0$ electron resonance,

$$
P=-\frac{i \omega}{8 \pi}\left[\left|E_{y}\right|^{2} \Delta K_{Y y}-2 i\left|E_{y} E_{z}\right| \sin \alpha \Delta K_{y z}+\left|E_{z}\right|^{2} \Delta K_{z z}\right]
$$

where we have made use of the onsager relation $\mathrm{K}_{\mathrm{yz}}=-\mathrm{K}_{\mathrm{zy}}$, and where

$$
\begin{aligned}
& \Delta \mathrm{K}_{\mathrm{YY}}=\frac{i}{\left.\right|_{\mathrm{Ce}} \mid} \frac{\mathrm{k}_{\mathrm{X}}^{2} \mathrm{~s}^{2}}{\omega} \mathrm{G} \\
& \Delta \mathrm{K}_{\mathrm{Yz}}=\frac{\mathrm{k}_{\mathrm{x}}}{\mathrm{k}_{\mathrm{z}}} \mathrm{G} \\
& \Delta K_{z z}=\frac{2 i \omega\left|\omega_{c e}\right|}{k_{z}^{2} s^{2}} G \\
& \mathrm{G} \equiv \pi^{1 / 2} \stackrel{\left.{ }^{(1)}\right)_{\mathrm{pe}}^{2}}{\left.\left.\right|_{\mathrm{ce}}\right\rceil} \frac{1}{\left.\right|_{\mathrm{k}} \mid \mathrm{s}} \exp \left(-\frac{\omega^{2}}{\mathrm{k}_{\mathrm{z}}^{2} \mathrm{~s}^{2}}\right), \\
& s^{2} \equiv \frac{2 k T_{e}}{m_{e}}, \quad \frac{E_{y}}{E_{z}}=\left|\frac{E_{y}}{E_{z}}\right| e^{i \alpha}
\end{aligned}
$$

The evaluation of the amplitude and phase relations between $E_{y}$ and $E_{z}$ is made in a straightforward fashion from the vector wave equation, Eq. (1), using the hot-electron forms for $\vec{K}$ including $\mathrm{K}_{\mathrm{yz}}$ and $\mathrm{K}_{\mathrm{zy}}$. The calculation is simplified in the 
weak-damping limit in that it suffices to retain only the reactive elements in $\vec{K}$. From the $x$ and $z$ components of the wave-equation one derives

$$
\frac{E_{y}}{E_{z}}=\frac{\left(n_{x} n_{z}\right)^{2}-\left(k_{x x}-n_{z}^{2}\right)\left(k_{z z}-n_{x}^{2}\right)}{-n_{x} n_{z} k_{x y}+\left(k_{x x}-n_{z}^{2}\right) k_{z y}}
$$

$\mathrm{K}_{\mathrm{xx}}$ and $\mathrm{K}_{\mathrm{xy}}$ involve mainly ions and the cold-plasma forms may be used. $k_{z z}=-\omega_{p e^{2}}^{2} \omega^{2}$ or $\left(k_{z}^{2} \lambda_{D}^{2}\right)^{-1}$ when $\left(\omega / k_{n} s\right)^{2} \gg 1$ and $\left(\omega / k_{n} s\right)^{2} \ll 1$ respectively, and $k_{z y}=0$ or $i k_{x} \omega_{p e}^{2} /\left(k_{z} \omega / \omega_{c e} \mid\right)$ in the same two limits respectively. If desired, one may use $B_{z}=\left(k_{x} c / \omega\right) E_{y}$ to express the transit-time damping in terms of the wave $B_{z}-f i e l d$.

Eq. (13) is of interest to us in this application only when Landau or transit-time damping are not negligible: In such regimes $\left(\omega / k_{n} s\right) \leqslant 1$, and the $k_{z y}$ term in the denominator will dominate. Then we may reasonably approximate

$$
\frac{E_{y}}{E_{z}} \simeq-\frac{K_{z z}}{K_{z y}}=i \frac{w e B}{k_{x} k_{z} T_{e} c}
$$

For low frequencies, a simple argument gives the same result. Say ions move in and out wi.th $\vec{B}$ - then by quasineutrality, $\mathrm{n}_{\mathrm{e}} \sim \mathrm{B}_{\mathrm{z}}$. Also, for hot electrons, $\mathrm{n}_{\mathrm{e}}=\mathrm{n}_{\mathrm{o}} \exp \left(\mathrm{e} \phi / \mathrm{kT}_{\mathrm{e}}\right)$. Finally, using the induction equation, $\partial \mathrm{B}_{\mathrm{z}} / \partial \mathrm{t}=-\mathrm{c} \partial \mathrm{E}_{\mathrm{y}} / \partial \mathrm{x}$, the above relation follows.

It is particularly interesting to observe that the substitution of this phase and amplitude relationship for $E_{y}$ and $E_{z}$ leads to exact cancellation between the transit-time term $\left(\Delta \mathrm{K}_{\mathrm{y}}\right)^{\prime}$ and the cross-term $\left(\Delta \mathrm{K}_{\mathrm{yz}}\right)$, leaving only the Landau damping term $\left(\Delta \mathrm{K}_{\mathrm{zz}}\right)$ which is then equal in magnitude to one-half of the 
transit-time term,

$$
P_{\text {total }} \simeq-\frac{i \omega}{16 \pi} \Delta K_{y y}\left|E_{y}\right|^{2}
$$

\section{WAVE ENERGY DENSSITY}

A convenient way to compute the wave damping rate is to divide the power absorption by the wave energy density, $W$. From conventional wave theory,

$$
W=\frac{1}{16 \pi}\left[\vec{B}^{*} \cdot \overrightarrow{B^{*}}+E^{*} \cdot \frac{\partial}{\partial \omega}\left(\omega \vec{K}_{h}\right) \cdot \vec{E}\right]
$$

where $\overrightarrow{\mathrm{K}}_{\mathrm{h}}$ is the Hermitian portion of the dielectric tensor. With the cold-plasma dielectric tensor elements, and making use of the phase relation between $E_{X}$ and $E_{Y}$ derived from Eq. (1), we find

$$
\begin{aligned}
& \mathrm{w}=\frac{1}{16 \pi} \cdot \frac{4 \pi \mathrm{n}_{i} \mathrm{~m}_{i} \mathrm{c}^{2}}{\mathrm{~B}^{2}}\left\{\mathrm{~N}_{\mathrm{n}}^{2}\left(\mathrm{H}^{2}+1\right)+\mathrm{N}_{\perp}^{2}\right. \\
& \left.+\frac{A}{2}^{2}\left[(1+H)^{2}\left(1-\frac{\omega}{\omega_{C i}}\right)^{2}+(1-H)^{2}\left(1+\frac{\omega}{\omega_{C i}}\right)^{2}\right]\right\}\left|E_{y}\right|^{2} \text { ， } \\
& H \equiv \frac{i E_{x}}{E_{y}}=-\frac{\omega_{C i}}{\omega} \cdot \frac{N_{\perp}^{2}+N_{11}^{2}-A}{A}, A \equiv \omega_{C i}^{2} /\left(\omega_{C i}^{2}-\omega^{2}\right)
\end{aligned}
$$

$\mathrm{N}_{\prime \prime}^{2}$ and $\mathrm{N}_{\perp}^{2}$ are related, of course, by the dispersion relation in Eq. (3). The first two terms inside the brackets represent magnetic field energy, and the $A^{2}$ term is the kinetic energy of the coherent particle motion.

\section{AN EXAMPLE}

To illustrate the calculations presented here, we look at one renresentative case, a tokamak plasma with the following parameters: 
Triton density

Deuteron density

Temperature

Magnetic field

Major radius

Minor radius

Wave frequency

Parallel wavelength
$\mathrm{n}_{\mathrm{T}}=5 \times 10^{13} \mathrm{~cm}^{-3}$

$\mathrm{n}_{\mathrm{D}}=2.5 \times 10^{12} \mathrm{~cm}^{-3}$

$\mathrm{kT}_{\mathrm{i}}=\mathrm{kT}_{\mathrm{e}}=4000 \mathrm{eV}$

$40 \mathrm{kG}$

$274 \mathrm{~cm}$

$60 \mathrm{~cm}$

$\omega=\omega_{C}$ (deuterons) $=2 \pi(30.5) \mathrm{MHz}$

$\lambda_{\text {" }}=123 \mathrm{~cm}$

The first group of computations concerns the background plasma, and the deuteron contribution is neglected.

$$
\begin{aligned}
& \frac{4 \pi n_{i} m_{i} c^{2}}{B^{2}}=1770 . \\
& \left(2 \mathrm{kT}_{\mathrm{e}} / \mathrm{m}_{\mathrm{e}}\right)^{1 / 2}=3.75 \times 10^{9} \mathrm{~cm} / \mathrm{sec} \\
& \left(2 \mathrm{kT}_{i} / \mathrm{m}_{i}\right)^{1 / 2}=5.06 \times 10^{7} \mathrm{~cm} / \mathrm{sec} \\
& \left(\omega / k_{n}\right)\left(2 k T e^{/ m_{e}}\right)^{-1 / 2}=1 \\
& \mathrm{n}_{\mathrm{z}}=7.99 \\
& \mathrm{~N}_{\prime \prime}^{2}=0.0362 \\
& \mathrm{n}_{\mathrm{x}}=39.6 \\
& \lambda_{\mathrm{x}}=2 \pi / \mathrm{k}_{\mathrm{x}}=24.8 \mathrm{~cm} \\
& \mathrm{~N}_{\perp}^{2}=0.886 \\
& \omega / \omega_{C}=1.50 \\
& A=-0.8 \\
& \mathrm{~K}_{\mathrm{xx}} \approx \mathrm{K}_{\mathrm{YY}}=-1410 \\
& \mathrm{~K}_{x y}=-\mathrm{K}_{\mathrm{yx}}=-\mathrm{i} 2120 \\
& \text { 'R }=707 \\
& L=-3530
\end{aligned}
$$




$$
\begin{aligned}
& \mathrm{K}_{z y}=-\mathrm{K}_{\mathrm{Yz}}=-3810-\mathrm{i} 445 \\
& \mathrm{~K}_{z z}=-6.60 \times 10^{5}+15.65 \times 10^{6} \\
& \Delta \mathrm{K}_{\mathrm{yY}}=\mathrm{i} 5.14 \\
& \Delta \mathrm{K}_{\mathrm{Yz}}=3810 \\
& \Delta \mathrm{K}_{\mathrm{zz}}=15.65 \times 10^{6} \\
& \mathrm{H}=1.435 \\
& \mathrm{~W}=65.0\left|\mathrm{E}_{\mathrm{Y}}\right|^{2} \quad(548 \text { magnetic, } 468 \text { kinetic }) \\
& \left|\mathrm{E}_{\mathrm{y}} / \mathrm{E}_{\mathrm{z}}\right|=1.453 \times 10^{3} \\
& \alpha=8.3 .4^{\circ} \\
& \mathrm{G}=3670 \\
& \mathrm{E}_{+} / \mathrm{E}_{-}=0.179 \\
& E_{+} / \mathrm{i} \mathrm{E}_{\mathrm{y}}=-0.218 \\
& \text { Volume }=1.947 \times 10^{7} \mathrm{~cm}^{3}
\end{aligned}
$$

The power computations show

$P_{\text {transit-time }}\left(\Delta \mathrm{K}_{\mathrm{YY}}\right)=3.92 \times 10^{7}\left|\mathrm{E}_{\mathrm{y}}\right|^{2} \mathrm{sec}^{-1}$

$P_{\text {cross-terms }}\left(\Delta \mathrm{K}_{\mathrm{yz}}\right)=-3.97 \times 10^{7}\left|\mathrm{E}_{\mathrm{y}}\right|^{2} \mathrm{sec}^{-1}$

$\mathrm{P}_{\text {Landau }}\left(\Delta \mathrm{K}_{\mathrm{zz}}\right)=2.04 \times 10^{7}\left|\mathrm{E}_{\mathrm{y}}\right|^{2} \sec ^{-1}$

$\mathrm{P}_{\text {Total }}$ (electrons) $=1.99 \times 10^{7}\left|\mathrm{E}_{\mathrm{y}}\right|^{2} \sec ^{-1}$

$Q($ electrons $) \equiv \omega W / P_{\text {total }}=628$

With the addition of $5 \%$ deuterons and electrons, both at $\mathrm{kT}=4000 \mathrm{eV}$, we compute

$\Delta \mathrm{R}$ (deuterons plus electrons) $=29.5$

$$
\begin{array}{ll}
\Delta \mathrm{L} \text { (electrons) } & =-59.0 \\
\Delta \mathrm{L} \text { (deuterons) } & =i 6330 .
\end{array}
$$

$E_{+} / E_{-}=0.0920 / 60.0^{\circ}$

$E_{+} / i E_{y}=0.0961 /-115.3^{\circ}$

We assume the central resonant surface passes through magnetic axis. 
Then $\sin \theta=1$ in Eq. (10), and the power absorbed per unit volume is

$$
\begin{aligned}
\mathrm{P}= & 8.32 \times 10^{6}(\mathrm{R} / \mathrm{r})\left|\mathrm{E}_{\mathrm{y}}\right|^{2} \mathrm{sec}^{-1} \\
\langle\mathrm{P}\rangle & \equiv\left(2 / \mathrm{a}^{2}\right) \int_{0}^{\mathrm{a}} \mathrm{P}(\mathrm{r}) \mathrm{rdr}, \text { for }\left|\mathrm{E}_{\mathrm{y}}\right|^{2}=\text { const, } \\
& =7.60 \times 10^{7}\left|\mathrm{E}_{\mathrm{Y}}\right|^{2}
\end{aligned}
$$

$\mathrm{Q}($ ions $)=\omega \mathrm{W} /\langle\mathrm{P}\rangle=164$

The kinetic energy of the deuterons has not been included in $W$ in the last expression; its inclusion will increase $Q$ (ions).

However, the small fraction of deuterons present and the unfavorable polarization of $\vec{E}$ will limit the size of this contribution.

\section{QUASILINEAR DIFFUSION COEFFICIENTS}

Generally in rf heating applications it is sufficient to know just the rate of power absorption from a wave of given amplitude. For creating a fusion-producing ion tail, however, we want to know precisely how the ion velocity distribution is affected. The means for this computation are readily available by including the quasilinear diffusion coefficient among the Fokker-Planck terms in the Boltzmann equation. The quasilinear diffusion coefficient for cyclotron absorption was calculated by Kennel and Engelmann [15], and in the absence of other diffusive processes, the kinetic ion equation reads

$$
\frac{\partial f(\vec{v})}{\partial t}=\lim _{V \rightarrow \infty} \sum_{n} \frac{\pi z^{2} e^{2}}{m^{2}} \int \frac{d^{3} \vec{k}}{(2 \pi)^{3} v} L \dot{v}_{\perp} \delta\left(\omega-k_{n} v_{n}-n \omega_{c j}\right)\left|\theta_{n, k}\right|^{2} v_{\perp} L f
$$

where

$$
L \equiv\left(1-\frac{k_{n} v_{n}}{\omega}\right) \frac{1}{v_{\perp}} \frac{\partial}{\partial v_{\perp}}+\frac{k_{11}}{\omega} \frac{\partial}{\partial v_{11}}
$$




$$
\begin{gathered}
\Theta_{n, k}=\frac{1}{2} e^{i \psi}\left(E_{x}-i E_{y}\right)_{k}^{J} J_{n+1}\left(\frac{k_{\perp} v_{\perp}}{\omega_{c i}}\right)+\frac{1}{2} e^{-i \psi}\left(E_{x}+i E_{y}\right)_{k} J_{n-1}\left(\frac{k_{\perp} v_{\perp}}{\omega_{c i}}\right) \\
+\frac{v_{1}}{v_{\perp}}\left(E_{z}\right)_{k} J_{n}\left(\frac{k_{\perp} v_{\perp}}{\omega_{c i}}\right) \\
\vec{k}=\hat{x} k_{\perp} \cos \psi+\hat{y} k_{\perp} \sin \psi+\hat{z} k_{\prime \prime}
\end{gathered}
$$

$V$ is the plasma volume, and $\vec{E}_{k}$ are the Fourier amplitudes in the usual complex analysis of the wave tield. For present purposes we can drop the parallel velocity effects in the $L$ operator, Eq. (18), but this point will be discussed at the end of this section. We may also neglect the $E_{z}$ and $E_{x}-i E_{y}$ (riqht-handed) contributions to $\theta_{n, k^{\prime}}$ assume $\psi=0$ and a monochromatic spectrum. Then, carrying out the integration in [18] over $\vec{k}$,

$$
\frac{\partial f(\vec{v})}{\partial t} \simeq \frac{\pi z^{2} e^{2}}{8 m^{2}\left|k_{n}\right|}\left|E_{x}+i E_{Y}\right|^{2} \sum_{n} \frac{1}{v_{\perp}} \frac{\partial}{\partial v_{\perp}} v_{l}^{2}\left|J_{n-I}\left(\frac{k_{\perp} v_{\perp}}{w_{C i}}\right)\right|^{2} \delta\left[v_{u}-\frac{\left(w-n \omega_{C i}\right)}{k_{n}}\right] \frac{1}{v_{\perp}} \frac{\partial f}{\partial v_{\perp}}
$$

summed over the cyclotron harmonics. The electric field in (19) is $\vec{E}=\operatorname{Re}\left(\hat{x}_{x}+\hat{y}_{y}\right) \exp (i \vec{k} \cdot \vec{r}-i \omega t)$. For acceleration at the cyclotron fundamental, the Bessel factor is $J_{0}\left(k_{\perp} v_{\perp} / \omega_{c i}\right) \rightarrow 1$ in the small Larmor-radius limit. To recover our previous result, Eq. (8), on ion heating, we simply integrate twice by parts,

$$
2 \pi \int v_{\perp} d v_{\perp} d v_{n} \frac{m v^{2}}{2} \frac{\partial f}{\partial t}=2 \pi \int v_{\perp} d v_{\perp}\left[\frac{\pi z^{2} e^{2}}{4 m\left|k_{n}\right|}\left|E_{x}+i E_{y}\right|^{2} f\left(v_{n}^{(r e s)}\right)\right]
$$

For comparison with (8) and (10), we observe that $\left|E_{x}+i E_{y}\right|^{2}=4\left|E_{+}\right|^{2}$. 
Two points of general interest now come up which concern the use of the quasilinear diffusion coefficients for the cyclotron heating calculation. The first, already referred to in Section 4 , is that the direct calculation of resonant-ion heating in the inhomogeneous magnetic field leads to the identical result, Eq. (8), as the local use of the quasilinear diffusion coefficient, Eq. (20), and that this identity of results therefore justifies the use of this coefficient even though the electric field spectrum is monochromatic. The critical consideration is that the usual quasilinear calculation demands a spectrum of randomly phased modes to justify the concept of true particle diffusion resulting from a number of incoherent velocity displacements; whereas in the present heating calculation we consider that the irradiation is monochromatic in both frequency and wavenumber. The usual problem for the monochromatic spectrum is that wave-particle phase relations can be altered by the very coherence of the wave, as when trapping occurs. However, this difficulty is easily avoided in the tokamak heating provess under consideration. In each passage through the resonant surface the particles here pick up small increments to their perpendicular velocity and to justify the description of this process as velocity-space diffusion we require just that successive velocity increments be randomly phased, i.e., that $u(-\infty)$ in the section 4 calculation be randomly phased with respect to the oscillating electric field. The necessary randomness easily appears from collisions. - the collision rate need only be such that particles "forget" the phase of the cyclotron heating field between successive transits of the resonant surface. Typically the time between successive transits will be the time for an ion to 
execute half a poloidal rotation in a tokamak, $\tau_{p} \approx \pi R q / v_{n}$. The irreproducibility in $\tau_{p}$ will be $\Delta \tau_{p} \approx \pi R q\left(\Delta v_{n}\right) / v_{n}^{2}$, and dispersion by coulomb scattering will lead to rms $\Delta v_{\text {n }}$ values of order $\left(\left\langle\left(\Delta v_{.,}\right)^{2}\right\rangle \tau_{p}\right)^{1 / 2}$ after half a poloidal rotation. Requiring that ions "forget" the cyclotron phase between transits just says that $\omega_{c i} \Delta \tau_{p}>1$, or that

$$
\left\langle\left(\Lambda v_{n_{i}}\right)^{2}\right\rangle \quad \therefore \therefore \frac{1}{\left(\omega_{c i}{ }^{\tau} p\right)^{2}} \frac{1}{\tau_{p}}
$$

The diffusion coefficient $\left\langle\left(\Delta v_{n}\right)^{2}\right\rangle$ is evaluated in

Eq. (28) in this paper, and the randomization criterion can be seen to be very easily satisfied in actual tokamaks, even in the ion banana regime.

The second point of general interest in this quasilinear diffusion calculation is that cyclotron heating is generally considered a process which causes dispersion only in $v_{\perp}$. However, a look at the structure of the diffusion operator, $L$, shows that dispersion in parallel velocities is also involved. In fact, the I operator is a gradient operator in velocity space directed along the circle

$$
v_{\perp}^{2}+\left(v_{n}-\frac{\omega}{k_{11}}\right)^{2}=\text { constant }
$$

and quasilinear diffusion takes place only along these circles, Fig. 4. The sketch illustrates that the amount of parallel diffusion occurring under cyclotron resonance can be appreciable. It must be remembered, however, that resonant particles are involved, so the diffusion occurs on the circles only where they intersect the line 
$v_{n}=\left(\omega-n \omega_{c i}\right) / k_{n}$, and the slope of the curves away from this intersection does not have physical meaning.

\section{FOKKER-PLANCK EQUATION}

We now want to write down a kinetic equation for the ion velocity distribution incorporating the combined effects of quasilinear heating and coulomb thermalization. A convenient form for the Fokker-Planck equation is

$$
\frac{\partial f}{\partial t}=-\nabla_{v} \cdot(\langle\Delta \vec{v}\rangle f)+\frac{1}{2} \nabla_{v} \cdot\left[\nabla_{v} \cdot(\langle\Delta \vec{v} \Delta \vec{v}\rangle f)\right]
$$

the first term representing drag, the second term dispersion. We will be satisfied to examine the evolution of the distribution function for a. small number of ions $(\mathrm{e} . \mathrm{g}$. , a small minority component of deuterons in a thermal triton plasma, or the high-energy nonMaxwellian ion tail thermalizing against an approximately Maxwellian ion body distribution) and ask only for the Fokker-planck coefficients for test particles diffusing in an isotropic Maxwellian plasma of ions and electrons. The needed coulomb coefficients are those of Chandrasekhar and Spitzer [16], given for Cartesian coordinates in velocity space as $\left\langle\Delta v_{11}\right\rangle,\left\langle\left(\Delta v_{n}\right)^{2}\right\rangle$ and $\left\langle\left(\Delta v_{1}\right)^{2}\right\rangle$ : (For these coulomb coefficients, $\perp$ and "denote directions with respect to the test-particle $\vec{v}$, and do not pertain to $\vec{B}$.) Going to spherical coordinates in velocity space and adding the quasilinear diffusion to the coulomb terms [17], the total kinetic equation is 


$$
\begin{aligned}
& \frac{\partial f}{\partial t}=C(f)+Q(f) \\
& \left.C(f) \equiv-\frac{l}{v^{2}} \frac{\partial}{\partial v}\left[v^{2}\left(\left\langle\Delta v_{n}\right\rangle+\frac{1}{2 v}<\left(\Delta v_{\perp}\right)^{2}\right\rangle\right) f\right] \\
& +\frac{1}{2 v^{2}} \frac{\partial^{2}}{\partial v^{2}}\left(v^{2}<\left(\Delta v_{n}\right)^{2}>f\right) \\
& +\frac{1}{4 v^{2}} \frac{\partial}{\partial \mu}\left(1-\mu^{2}\right) \frac{\partial}{\partial \mu}\left(<\left(\Delta v_{\perp}\right)^{2}>f\right) \\
& Q(f) \equiv \sum_{m n} Q_{m n} \delta\left(\omega_{c i}-\frac{\left(\omega-k_{11} \nabla_{l 1}\right)}{n}\right) R_{m}(f) \\
& R_{m}(f) \equiv\left(1-\mu^{2}\right)^{m+1} \frac{1}{v^{2}} \frac{\partial}{\partial v} v^{2 m+1} \frac{\partial}{\partial v} v f+v^{2 m-2} \frac{\partial}{\partial \mu} \mu\left(1-\mu^{2}\right)^{m+1} \frac{\partial}{\partial \mu} \mu f \\
& -v^{2 m-2} \frac{\partial}{\partial \mu} \mu\left(1-\mu^{2}\right)^{m+1} \frac{\partial}{\partial v} v f \\
& -\left(1-\mu^{2}\right)^{m+1} v^{2 m-2} \frac{\partial}{\partial \mu} \mu \frac{\partial}{\partial v} v f-2 m\left(1-\mu^{2}\right)^{m+1} v^{2 m-2} \frac{\partial}{\partial \mu} \mu f,
\end{aligned}
$$


Since $\omega_{c i}$ is a function of the major radius in a tokamak, we can eliminate the delta function in Eq. (22) by averaging over a toroidal magnetic surface of minor radius $r$, major radius $R$, as in the derivation of Eq. (10);

$$
\begin{aligned}
& \frac{1}{2 \pi} \int \mathrm{d} \theta \mathrm{Q}_{\mathrm{mn}}(\mathrm{x}, \mathrm{y}) \delta\left[\omega_{\mathrm{ci}}-\frac{\left(\omega-\mathrm{k}_{n} \mathrm{v}_{n}\right)}{\mathrm{n}}\right] \\
& \approx \frac{\mathrm{R}}{\pi(1) \mathrm{ci}^{r\left|\sin \theta_{\mathrm{o}}\right|}} \mathrm{Q}_{\mathrm{mn}}\left(\mathrm{x}_{\mathrm{o}},\left|\mathrm{y}_{\mathrm{o}}\right|\right)
\end{aligned}
$$

where $\theta_{0}=\tan ^{-1}\left(\left|y_{0}\right| / x_{0}\right)$, and $x_{0}, y_{0}$ are the coordinates in the plasma cross-section where the selected magnetic surface intersects the central $\left(v_{n}=0\right)$ resonant surface. Compare Fig. 3. We have assumed that the cross-section of the magnetic surface is a circle centered around $(x, y)=(0,0)$, that. $f=f(r, \vec{v})$, independent of the spatial angle $\theta$, and that $Q_{m n}(x, y)$ is symmetric for $y= \pm\left|\dot{y}_{0}\right|$ and is well approximated over the resonant interaction region by its central value at $\mathbf{x}=\mathbf{x}_{0}$.

\section{EXPANSION OF $f(\vec{v})$ IN LEGENDRE POLYNOMIALS}

The conventional way to obtain an analytic solution to a partial differential equation such as Eq. (22) is by the expansion. of $f$ in a series of Legendre polynomials in $\mu$,

$$
f(r, \vec{v}, t)=\sum_{\ell=0}^{\infty} g_{2 \ell}(r, v, t) P_{2 \ell}(\mu)
$$

For $Q(f)=0$, the expansion is particularly appropriate since the $P_{k}(\mu)$ are eigenfunctions of the $C(f)$ operator. $Q(f) \neq 0$ 
unfortunately mixes the various $P_{k}(\mu)$ 'functions, but the expansion is still useful because the $\mu$ integrations are easy, and the problem is quickly reduced to a set of coupled linear differential equations in $v$ and $t$. Restricting our attention to the case for heating at the fundamental cyclotron frequency $(n=1)$, and keeping only the first two terms in the $\mu$-expansion,

$$
f(v, t)=A(v, t)+\frac{1}{2} B(v, t)\left(3 \mu^{2}-1\right),
$$

we obtain from the $P_{0}(\mu)$ and $P_{2}(\mu)$ moments of Eq.

$$
\begin{aligned}
\frac{\partial A}{\partial t}= & \frac{1}{v^{2}} \frac{\partial}{\partial v}\left[-\alpha v^{2} A+\frac{1}{2} \frac{\partial}{\partial v}\left(B v^{2} A\right)\right. \\
& \left.+K v \frac{\partial}{\partial v} v\left(A-\frac{B}{5}\right)-K\left(A+\frac{2}{5} B\right) v\right] \\
\frac{\partial B}{\partial t}= & -\frac{1}{v^{2}} \frac{\partial}{\partial v}\left(\alpha v^{2} B\right)+\frac{1}{2 v^{2}} \frac{\partial^{2}}{\partial v^{2}}\left(B v^{2} B\right)-\frac{3}{2} \frac{\gamma}{v^{2}} B \\
& +\frac{K}{v^{2}} \frac{\partial}{\partial v} v \frac{\partial}{\partial v} v\left[-A+\frac{5}{7} B\right]-\frac{K}{v^{2}}\left[3 A+\frac{30}{7} B\right] \\
& +\frac{K}{v^{2}} \frac{\partial}{\partial v} v\left[4 A-\frac{5}{7} B\right]
\end{aligned}
$$

in which, using Eqs. (10), (23), and (24), 


$$
\begin{aligned}
& K \equiv \frac{2 R}{3 \pi \omega_{c i} \mid \sin \theta_{0}} Q_{01}\left(x_{0},\left|y_{0}\right|\right)=\frac{\langle P\rangle}{3 n_{i} m_{i}}, \\
& \alpha \equiv\left\langle\Delta v_{n}\right\rangle+\frac{1}{2 v}\left\langle\left(\Delta v_{\perp}\right)^{2}\right\rangle, \\
& \beta \equiv\left\langle\left(\Delta v_{n}\right)^{2}\right\rangle \\
& \gamma \equiv\left\langle\left(\Delta v_{\perp}\right)^{2}\right\rangle
\end{aligned}
$$

\section{THE COULOMB DIFFUSION COEFFICIENTS}

The coulomb diffusion coefficients are given in spitzer [16]. We use subscript $f$ to designate the background-plasma field particles, ions and electrons,

$$
\begin{aligned}
& \left\langle\Delta v_{11}\right\rangle=-\sum_{f} C_{f} \ell_{f}^{2}\left(I+\frac{m}{m_{f}}\right) G\left(\ell_{f} v\right), \\
& \left\langle\left(\Delta v_{n}\right)^{2}\right\rangle=\sum \frac{C_{f}}{v} G\left(l_{f} v\right) \quad, \\
& \left\langle\left(\Delta v_{\perp}\right)^{2}\right\rangle=\sum_{f} \frac{C_{f}}{v}\left[\Phi\left(l_{f} v\right)-G\left(l_{f} v\right)\right], \\
& c_{f} \equiv \frac{8 \pi n_{f} z_{f}^{2} z^{2} e^{4} \ln \Lambda}{m^{2}} \text {. } \\
& \ell_{f}^{2} \equiv m_{f} / 2 k T_{f} \\
& \Phi(x) \equiv \frac{2}{\pi / 2} \int_{0}^{x} \exp \left(-y^{2} d y\right), \\
& G(x) \equiv \frac{\Phi(x)-x \Phi^{\prime}(x)}{2 x^{3}}
\end{aligned}
$$

In working with these diffusion coefficients, we will find the following identity useful, 


$$
-\alpha v^{2}+\frac{1}{2} \frac{d}{d v}\left(\beta v^{2}\right)=\sum_{f} C_{f} \frac{m v^{2}}{2 k T_{f}} G\left(l_{f} v\right)
$$

In addition, we introduce simple approximations to the somewhat cumbersome $G$ and $\Phi$ functions. Both approximations produce the correct leading terms in the two limits of small and large $x$; in midrange, at the points of maximum fractional error, $\mathrm{G}(1.819)$ is about $10.5 \%$ too low, while $\Phi(1.040)$ is about 27. 4 \% too high. The two approximating forms are

$$
\begin{aligned}
G(x) & : \approx \varepsilon x /\left(1+2 \varepsilon x^{3}\right) \\
\Phi(\bar{x}) & \approx \varepsilon\left(3 x+2 x^{3}\right) /\left(1+2 \varepsilon x^{3}\right), \\
\varepsilon & \equiv 2 / 3 \pi^{1 / 2}
\end{aligned}
$$

It will be seen shortiy that the use of these special rums permits the complete solution of the one-dimensional Fokker-Planck equation by elementary integrals. Meanwhile, the following more easily interpreted forms for the coulomb diffusion coefficients may be derived from the preceding equations using just the leading terms for $G(x)$ and $\Phi(x)$,

$\underline{\alpha}$

Range I

$\mathrm{D} / \mathrm{v}$

Range II $-\frac{v}{t_{s}}\left(I+\frac{v_{\alpha}^{3}}{v^{3}}\right)$ $\underline{\beta}$

D

$$
\frac{2 k T_{e}}{m t_{s}}\left(1+\frac{v_{\beta}^{3}}{v^{3}}\right)
$$

$\underline{Y}$

$2 \mathrm{D}$ 
where Range $I$ is for $v \ll\left(2 \mathrm{kT}_{\mathrm{i}} / \mathrm{m}_{\mathrm{i}}\right)^{1 / 2}$ and Range II for $\left(2 \mathrm{kT}_{i} / \mathrm{m}_{i}\right)^{1 / 2} \ll \mathrm{v} \ll\left(2 \mathrm{kT}_{\mathrm{e}} / \mathrm{m}_{\mathrm{e}}\right)^{1 / 2}$, and where, summing over the $f$ species of field ions and electrons,

$$
\begin{aligned}
D & \equiv \varepsilon \sum_{f} C_{f_{f} l_{f}} \\
& =2 \sum_{f} \frac{n_{f} z_{f}^{2}}{n_{e}}\left(\frac{m_{f} T_{e}}{m_{e} T_{f}}\right)^{1 / 2} \frac{k T e}{m_{s}}, \\
t_{S} & \equiv \frac{m_{e}}{\varepsilon m c_{e}}\left(\frac{2 k T_{e}}{m_{e}}\right)^{3 / 2} \\
& =6.27 \times 10^{8} \frac{A\left(k T_{e}\right)^{3 / 2}}{z^{2} n_{e} \ln \Lambda} \text { seconds. }
\end{aligned}
$$

Summing over the $j$ species of field ions,

$$
\begin{aligned}
& \frac{1}{2} m v_{\alpha}^{2}=14.8 \mathrm{kT} e^{\left[\frac{A^{3 / 2}}{n_{e}} \sum_{j} \cdot \frac{n_{j} z_{j}^{2}}{A_{j}}{ }^{2 / 3}\right.}, \\
& \frac{1}{2} m v_{B}^{2}=14.8\left(\mathrm{kT}_{e}\right)^{1 / 3}\left[\frac{A^{3 / 2}}{n_{e}} \sum_{j} \frac{n_{j} z_{j}^{2} k_{j}}{A_{j}}{ }^{2 / 3},\right. \\
& \frac{1}{2} m v_{\gamma}^{2}=14.8 \mathrm{kT}_{e}\left[\frac{2 A^{1 / 2}}{n_{e}} \sum_{j} n_{j} z_{j}^{2}\right]^{2 / 3}
\end{aligned}
$$

$A$ and $A_{j}$ are the atomic masses of the test and field ions, $n_{e}$ is in $\mathrm{cm}^{-3}$, and $\mathrm{kT} e$ in $\mathrm{eV}$.

\section{STEADY-STATE $f(v, \mu)$, LOWEST ENERGY RANGE}

Now armed with explicit forms for the diffusion coefficients, we can look for solutions to the Fokker-Planck equation for the 
rf-heated resonant ions. In most rf-heating experiments, the experimentalist is able to irradiate the plasma with an rf pulse of sufficient duration that the temperatures reach a steady-state value or, due to impurity influx, may even start to droop. It is meaningful then to look for steady-state solutions to Eq. (22) or Eqs. (25) and (26). It should be noted, however, that the occurrence of a steady-state solution is peculiar to heating at

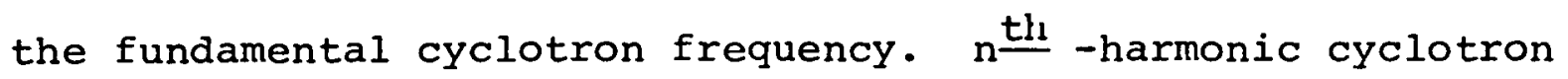
heating would replace $K$ in the integral of Eq. (31) by a quantity proportional to $\mathrm{Kv}^{2 n}+\ldots$, as required by Eqs. (22) and (23), and the calculated distribution function will no longer give convergent number and energy densities. Physically, harmonic heating acts preferentially on the higher-energy, larger-Larmorradius particles, and if unchecked, leads to time-dependent runaway of their energy.

For thermal energies and above, the full determination of $\mathrm{f}(\mathrm{V}, \mu)$ probably requires computer analysis, but in the lowest energy range, i.e., for $v \ll\left(2 \mathrm{kT}_{i} / \mathrm{m}_{i}\right)^{1 / 2}$, the diffusion coefficients are sufficiently simple that an explicit solution to Eqs. (25) and (26) is easily obtained. In steady-state, $\partial A / \partial t=0$ and Eq. (25) can be integrated one time immediately. Furthermore, the condition that $A$ and its derivatives vanish as $v \rightarrow \infty$ requires that the constant of integration be zero. Then using identity (29) and the Range I approximations for $\alpha, \beta$ and $\gamma$, we find. 


$$
\begin{aligned}
& A=\text { constant } \exp -\left(\mathrm{mv}^{2} / 2 \mathrm{kT} \mathrm{eff}_{\mathrm{f}}\right), \\
& k T_{\text {eff }}=(D+2 K) /\left[\varepsilon \sum_{f}\left(C_{f} \ell_{f} / k T_{f}\right)\right] \\
& B=\frac{4 K}{7 D+10 K}\left(\frac{\mathrm{mv}^{2}}{2 k T \text { eff }}\right)^{2} A
\end{aligned}
$$

The f-sum, again, is over background ions and electrons. When all $\mathrm{T}_{\mathrm{f}}$ are equal, $\mathrm{T}_{\mathrm{f}}=\mathrm{T}_{\mathrm{o}^{\prime}}$ then $\mathrm{kT}$ eff $=(\mathrm{D}+2 \mathrm{~K}) \mathrm{kT} / \mathrm{D}$. And further, when $K \rightarrow 0$, then $T_{\text {eff }} \rightarrow T_{O}$ and $B \rightarrow 0$. When $K \neq 0$, the resonant-ion velocity distribution is still approximately Maxwellian for the lowest range of velocities, but with an effective temperature above that of the background ions and electrons. A curious artifact of this $K \neq 0$. solution is that the constant$f(\vec{v})$ surfaces near the origin of velocity space are prolate spheroids around the $\vec{B}$ direction even though the applied wave-diffusion introduces dispersion in $v_{\perp}$. Were it not for a certain cancellation of terms in Eq. (26) when $A$ is of the Maxwellian form, the lowest term for $B$ would be $B \sim-v^{2} A$ and the corresponding constant-f( $(\vec{v})$ surfaces would be the expected oblate spheroids. As indicated earlier, the complexity of the diffusion coefficients makes the full determination of " $f(v, \mu)$ much more difficult at thermal energies and above. Nevertheless, a very useful estimate of the magnitude of the angular dependence may be obtained by integration of Eq. (26) over $\mathrm{v}^{2} \mathrm{dv}$ from 0 to $\infty$. The only surviving terms are

$$
\int_{0}^{\infty}\left(\frac{\gamma(v)}{2}+\frac{10}{7} K\right) B(v) d v=-K \int_{0}^{\infty} A(v) d v
$$


which suggests that

$$
B(v) \sim-K A(v) /\left[\frac{\gamma(v)}{2}+\frac{10}{7} K\right],
$$

as a very rough estimation. We note that the indicated ellipticity is, happily in this case, oblate, proportional to $\mathrm{K}$ for small $\mathrm{K}$, and that $\mathrm{B} / \mathrm{A}$ approaches a constant negative value for large $\mathrm{K}$. (The expansion in Legendre poloynomials for the extreme case of 'a thin-disc distribution function, $f(\vec{v}) \sim \delta(\mu)$, would lead to the value $-5 / 4$ for the ratio of the coefficient of $\mathrm{F}_{2}(\mu)$ to that of $\left.\mathrm{P}_{0}(\mu).\right)$

The use of just two terms in the $\mathrm{P}(\mu)$ expansion is a rather unsatisfactory way to represent the angular dependence of $f(\vec{v})$ when $K$ is, large. Additional terms could be included, at the obvious cost of increasing analytic complexity, but for the large-K case it is worthwhile to explore the alternative representation of the Fokker-Planck equation using $v_{1}, v_{n}$ as variables rather

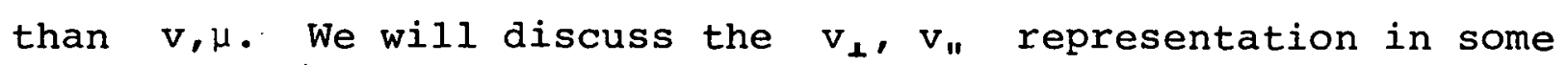
detail in section 14 .

\section{STEADY-STATE $f(v)$ SOLUTION}

The determination of the resonant-ion velocity distribution function is impressively simplified if we drop angular dependence from the model. The expansion of the Fokker-Planck equation, truncated at $f(\vec{v})=A(v)=(1 / 2) \int f(v, \mu) d \mu$, is then just Eq. (25) with $B=0$ and it is immediately integrable,

$$
f(v)=f(0) \exp -\int_{0}^{v} d v \frac{-2 \alpha v^{2}+\left(\beta v^{2}\right)^{\prime}}{\beta v^{2}+2 K v^{2}}
$$


The Coulomb diffusion coefficients $\alpha$ and $\beta$ are given in (27) and (28), and identity (29) provides a convenient form for the numerator of the integrand. For the background electron contributions to $G(x)$ it suffices to use $G\left(x_{e}\right) \approx \varepsilon x_{e}$ since in the velocity range of interest $v \ll\left(2 \mathrm{kT}_{\mathrm{e}} / \mathrm{m}_{\mathrm{e}}\right)^{1 / 2}$. We assume $a$ single species of background ions with density, temperature and charge $n_{j}, T_{j}$, and $z_{j} e$, and use the approximating form for $G\left(x_{j}\right)$ offered in $(30)$. Algebraic manipulation of the integrand then leads to the explicit solution for $f(v)$,

$$
\begin{aligned}
& \ln f(v)=-\frac{E}{k T_{e}(1+\xi)}\left[1+\frac{R_{j}\left(T_{e}-T_{j}+\xi T_{e}\right)}{T_{j}\left(1+R_{j}+\xi\right)} H\left(E / E_{j}\right)\right] \\
& E \equiv m v^{2} / 2, \quad \ell_{j} \equiv\left(m_{j} / 2 \mathrm{kT}_{j}\right)^{1 / 2}, \\
& R_{j} \equiv n_{j} z_{j}^{2}{ }_{j} / n_{e}^{l} e^{\prime}, \varepsilon \equiv 2 / 3 \pi^{1 / 2},
\end{aligned}
$$

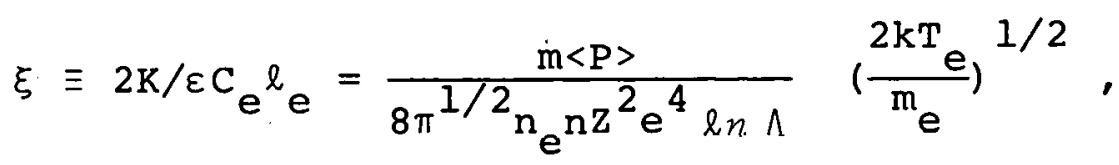

$$
\begin{aligned}
& E_{j}(\zeta)=\frac{m^{k T} i_{j}}{m_{j}}\left[\frac{1+R_{j}+\xi}{2 \varepsilon(1+\xi)}\right]^{2 / 3} \leqslant E_{j}(0), \\
& E_{j}(0)=\frac{1}{2} m v_{B}^{2}, \text { cf. Eq. (31), } \\
& H(x) \equiv \frac{1}{x} \int_{0}^{x} \frac{d u}{1+u^{3 / 2}} \\
& =\frac{2}{x}\left[\frac{1}{6} \ln \frac{1-x^{1 / 2}+x}{1+2 x^{1 / 2}+x}+\frac{1}{3^{1 / 2}}\left(\frac{\pi}{6}+\tan ^{-1} \frac{2 x^{1 / 2}-1}{3^{1 / 2}}\right)\right] \\
& \approx 1-\frac{2}{5} x^{3 / 2}+\frac{1}{4} x^{3}-\frac{2}{11} x^{9 / 2}+\ldots \\
& \approx 2.4184 x^{-1}-2 x^{-3 / 2}+\frac{1}{2} x^{-3}-\frac{2}{7} x^{-9 / 2}+\ldots
\end{aligned}
$$


Intermediate pairs of values for $x, H(x)$ are $0.1,0.9876$;

$0.2,0.9661 ; 0.4,0.9124 ; 0.7,0.8263 ; 1,0.7471 ; 2,0.5544 ;$

$4,0.3619 ; 7,0.2389$, and $10,0.1791$.

$\mathrm{Z}, \mathrm{m}, \mathrm{n}$ and $\mathrm{v}$ designate the charge number, mass, density and velocity of the resonant (test) ions. The effect of wave heating appears through the single dimensionless parameter $\xi$ which is directly proportional to $\langle\mathrm{P}\rangle$, the wave-heating power per unit volume delivered to the test ions, averaged over a magnetic surface.

To discuss the behavior of $f(v)$ in Eq. (34), it is useful to define an effective temperature at each value of $\mathrm{E}=\mathrm{mv}^{2} / 2$, $k \mathrm{~T}_{\text {eff }} \equiv-[\mathrm{d}(\ln \mathrm{f}) \cdot / \mathrm{dE}]^{-1}$,

$$
\frac{1}{k T_{\text {eff }}}=\frac{1}{k T_{e}(1+\xi)}\left[1+\frac{R_{j}\left(T_{e}-T_{j}+\xi T_{e}\right)}{T_{j}\left(1+R_{j}+\xi\right)} \cdot \frac{1}{1+\left(E / E_{j}\right)^{3 / 2}}\right] .
$$

For $\xi=0$ the body of the test-ion distribution is Maxwellian with temperature very close to that of the background ions ( $k T_{\text {eff }} \approx \mathrm{kT}_{j}$ ), while the tail of the test-ion distribution $\left(E \geqslant E_{j}\right)$ is influenced by the background electrons. For $\xi>0, k T$ eff for $E \ll E_{j}(\xi)$, identical. to $\mathrm{kT}_{\text {eff }}$ derived earlier in section 12, is somewhat above the background ion temperature. As $\mathrm{E}$ increases, the effective temperature increases and, for $E \gg E_{j}(\xi), k T$ eff approaches the asymptotic value $k \mathrm{~T}_{\mathrm{e}}(1+\xi)$ at which the waveinduced test-ion dispersion is entirely balanced by electron drag.

Plots of $\ln f(v)$ versus $E$ are shown in Fig. 5 for the parameters of the example introduced earlier, using $n_{j} z_{j}^{2} / n_{e}=3$ 
for a $z_{\text {eff }}=3$ in computing $R_{j}$. For these same parameters, the wave-induced dispersion level $\xi=100$ corresponds to

1.3 watts per $\mathrm{cm}^{3}$ rf power input to the plasma, averaged over a magnetic surface. Maintaining this average power level over the full volume of the model plasma in section 7 would require a total rf power input of 24 megawatts.

\section{STEADY-STATE $f\left(v_{\perp}\right)$ SOLUTION}

Our efforts to determine the angular dependence of $f(\vec{v})$ were fully successful only in the sub-thermal range of ion velocities, although the integral relation (32) provides a useful estimate of the anisotropy at thermal energies and above. However, at the very high energies in the tail of the two-component plasma, specifically, at test-ion energies above $\sim \mathrm{mv}_{\alpha}^{2} / 2$, the dominant Coulomb process is electron drag which does not introduce appreciable pitch-angle scattering. Of especial interest is that the perpendicular energy acquired from cyclotron heating is not shared with the parallel degree of freedom, so that the coefficient of wave-induced dispersion is increased by 508 throughout this energy range.

To describe the strongly anisotropic velocity distribution expected at high energies, it is fitting to change from $v, \mu$ coordinates in velocity space to $v_{1}, v_{11}$ coordinates. In the new variables, the coulomb terms in Eq. (22) are more intricate but the quasilinear term reverts to the simpler form seen in Eq. (19). The test-ion Fokker-Planck equation becomes 


$$
\begin{aligned}
& \frac{\partial f\left(v_{\perp}, v_{n}, t\right.}{\partial t}=-\frac{1}{v}\left(v_{\perp} \frac{\partial}{\partial v_{\perp}}+v_{n} \frac{\partial}{\partial v_{n}}+2\right)(\alpha f) \\
& +\frac{1}{2 v^{2}}\left(v_{\perp}^{2} \frac{\partial^{2}}{\partial v_{\perp}^{2}}+2 v_{\perp} v_{n} \frac{\partial^{2}}{\partial v_{\perp} \partial v_{n}}+v_{n}^{2} \frac{\partial}{\partial v_{n}^{2}}+4 v_{\perp} \frac{\partial}{\partial v_{\perp}}+4 v_{n} \frac{\partial}{\partial v_{n}}+2\right) \\
& +\frac{1}{4 v^{2}}\left(v_{n}^{2} \frac{\partial^{2}}{\partial v_{\perp}^{2}}-2 v_{\perp} v_{n} \frac{\partial^{2}}{\partial v_{\perp} \partial v_{n}}+v_{\perp}^{2} \frac{\partial^{2}}{\partial v_{n}^{2}}+\frac{v_{n}^{2}-v_{\perp}^{2}}{v_{\perp}}-2 v_{n} \frac{\partial}{\partial v_{n}}\right)(\gamma f) \\
& +\sum_{I I,, I I} Q_{m n} \delta\left(\omega_{C i}-\frac{\left(\omega-k_{n} v_{n}\right)}{n}\right) \frac{1}{v_{\perp}} \frac{\partial}{\partial v_{L}} v_{\perp}^{2 m+1} \frac{\partial f}{\partial v_{\perp}}
\end{aligned}
$$

$v \equiv\left(v_{\perp}^{2}+v_{n}^{2}\right)^{1 / 2}, Q_{m n}$ is defined in (23), and $\alpha, \beta$, and $\gamma$ are defined in (27). Equation (35) is equivalent to Eq. (22), but we now direct our attention to regions where the distortion is strong enough that $\left.\left.\left\langle v_{\perp}^{2}\right\rangle\right\rangle\right\rangle\left\langle v_{n}^{2}\right\rangle$, and order terms according to $v_{\perp}>\left|v_{n}\right|, \quad v_{\perp} \approx v_{1} \quad v_{n}\left(\partial / \partial v_{n}\right) \sim v_{\perp}\left(\partial / \partial v_{\perp}\right)$. We average Eq. over a magnetic surface, using (24), then define

$$
f\left(v_{1}\right) \equiv \int_{-\infty}^{\omega} f(\bar{v}) d v_{n}
$$

and integrate (35) over $v_{11}$. After some further straightforward integrations by parts (some small care must be taken with the $v_{\perp}^{2} \partial^{2}(\gamma f) / 4 v^{2} \partial^{2} v_{n}^{2}$ term), the dominant terms are found for a one-dimensional Fokker-Planck equation describing heating at the cyclotron fundamental frequency,

$$
\begin{aligned}
\frac{\partial f\left(v_{\perp}, t\right)}{\partial t} \approx & -\frac{1}{v_{\perp}} \frac{\partial}{\partial v_{\perp}}\left(\alpha v_{\perp} f\right)+\frac{1}{2 v_{\perp}} \frac{\partial^{2}}{\partial v_{\perp}^{2}}\left(\beta v_{\perp} f\right)+\frac{l}{4 v_{\perp}} \frac{\partial}{\partial v_{\perp}}(\gamma f) \\
& +\frac{3}{2} \frac{K}{v_{\perp}} \frac{\partial}{\partial v_{\perp}} v_{\perp} \frac{\partial f}{\partial v_{\perp}}
\end{aligned}
$$


Like Eq. (25), Eq. (36), with $\partial f\left(v_{1}, t\right) / \partial t=0$, can be quickly integrated twice to yield a steady-state solution,

$$
f\left(v_{\perp}\right)=f(0) \exp -\int_{0}^{v_{\perp}} d v_{\perp} \frac{-4 \alpha v_{\perp}+2\left(\beta v_{\perp}\right)^{\prime}+\gamma}{2 \beta v_{\perp}+6 k v_{\perp}} .
$$

Apart from the $50 \%$ increase in $K$, already discussed, Eq. (37) differs from its counterpart, Eq. (33), by the presence of the pitch-angle scattering coefficient in the numerator of the integrand. Pitch-angle scattering, like dispersion and drag, reduces $v_{\perp}$ when $\left.\left.v_{\perp}^{2}\right\rangle\right\rangle\left\langle v_{n}^{2}\right\rangle$.

Following the same steps which led from (33) to (34), making use in this instance as well of the approximating form for $\Phi(x)$ in $(30)$, we find

$$
\ln f\left(v_{\perp}\right)=\frac{-2 E}{k T_{e}(2+3 \xi)}\left\{1+\frac{R_{j}\left[\left(2 A+A_{j}\right)(2+3 \xi) T_{e}-4 A T_{j}\right]}{2 A T_{j}\left(2+2 R_{j}+3 \xi\right)} H\left(E / E_{j}\right)\right\}
$$

$$
E_{j} \equiv \frac{A k T_{j}}{A_{j}}\left[\frac{2+2 R_{j}+3 \xi}{2 \varepsilon(2+3 \xi)}\right]^{2 / 3}
$$

Apart from $E_{j}$, the quantities in (38) are defined as in (34). $A$ and $A_{j}$ are the atomic masses of the test and field ions, respectively.

It should be remembered that Eq. (38) only applies to the velocity range where $f(\vec{v})$ is strongly anisotropic. An estimate of the lower boundary for this range of $v_{1}$ can come from examining the numerator of the integrand in (37): the pitch-angle 
scattering term is smaller in magnitude than the electron drag . term when $v_{\perp}^{3}>v_{\gamma}^{3} / 4$, defined in Eq. (31). Above this value of test-ion velocity, where pitch-angle scattering becomes relatively weak, the main source of parallel energy to the distribution will be by its incidental transport as particles wave-diffuse upward in $v_{\perp}$ carrying their $v_{\|}$with them. The argument suggests that while the perpendicular distribution in the tail is described by (38), the parallel distribution for these same particles might be characterized by $\mathrm{kT}_{n} \sim \mathrm{mv}_{\gamma}^{3} / 8$.

\section{5. $f(v)$ FOR STEADY-STATE ISOTROPIC ION INJECTION}

It is interesting, for the purpose of comparison, to examine the velocity distribution which results from the Coulomb slowingdown and diffusion of a group of test ions injected at high energy (as energetic neutral atoms) into a tokamak plasma. We assume the injected beam is originally monoenergetic at $\mathrm{mv}^{2} / 2=\mathrm{w}$ and isotropic in its velocity distribution. 'l'o formulate a steadystate model, we also assume an ion sink at some much lower energy, $\mathrm{w}_{\mathrm{S}}$, which absorbs test ions at the same rate that the source emits them. The Fokker-Planck equation is again Eq. (25) with $\partial A / \partial t=0, B=0, \quad K=0$, and with a source term, $\sim \mathrm{v}^{-2} \cdot \delta\left[\mathrm{v}-(2 \mathrm{~W} / \mathrm{m})^{1 / 2}\right]$, and a sink term, $\sim-\mathrm{v}^{-2} \cdot \delta\left[\mathrm{v}-\left(2 \mathrm{w}_{\mathrm{s}} / \mathrm{m}\right)^{1 / 2}\right]$, added to the right-hand side. The first integration is immediate, the delta functions integrating into step functions; introduction of an integrating factor facilitates the second integration. Enormous simplification of the result occurs when the background 
ions and electrons have the same temperature, kT. Then $A_{0}=$ constant $\cdot \exp -m v^{2} / 2 \mathrm{kT}$ must satisfy the steady-state equation without sources or sinks, and this very solution in fact follows directly from $A_{0}=$ constant exp $-\int d v\left[2 \alpha v^{2}-\left(\beta v^{2}\right)^{\prime}\right] /\left(\beta \dot{v}^{2}\right)$ and Eqs. (27), (28) and (29). With source and sink as just described, the full solution in the range $w_{s}<\mathrm{mv}^{2} / 2<\mathrm{w}$ is

$$
f(v)=\text { constant } \cdot \exp -\frac{m v^{2}}{2 k T} \int d v\left(\exp \frac{m v^{2}}{2 k T}\right) / B v^{2}
$$

and use of the Range II approximation for $\beta$ then leads to

$$
f(v) \approx \text { constant } \cdot \exp -\frac{E}{k T} \int_{0}^{E} d U \frac{\exp (U / k T)}{1+\left[U / E_{j}(0)\right]^{3 / 2}}
$$

provided both $\mathrm{E} \equiv \mathrm{mv^{2 }} / 2>\mathrm{kT}$ and $\mathrm{W}_{\mathrm{S}}<\mathrm{E}<\mathrm{W}$. For $\mathrm{E}>\mathrm{W}$, the upper limit in the integral is $w \cdot E_{j}(0)=m v_{\beta}^{2} / 2$ is defined in Eqs. (3I) and (34). Moreover, for $\mathrm{kT}_{\mathrm{e}}=\mathrm{kT}_{\mathrm{j}}=\mathrm{kT}, \mathrm{Eq} \cdot$ (3I) shows $v_{\beta}^{2}=v_{\alpha}^{2}$, and provided the denominator in the integrand of (39) does not change significantly over a range of $U, \Delta U \sim \mathrm{kT}$, the integral may be approximated

$$
f(v) \approx \frac{\text { constant }}{v^{3}+v_{\alpha}^{3}}
$$

which is the customary solution for the distribution of originallymonoenergetic test ions, from a calculation based on drag alone and neglecting dispersion.

A plot of Eq. (39) for $200 \mathrm{keV}$ deuteron injection into a $4 \mathrm{keV}$ temperature tritium plasma is shown for comparison in Fig. 5. 


\section{EFFICIENCY FOR PRODUCTION OF FUSION REACTIONS}

A primary objective in two-component plasma research will be the production of abundant D-T nuclear reactions, hopefully matching or exceeding the rate per unit plasma volume required for an economic fusion reactor. Toward achieving this goal, rf heating can be helpful in heating the background plasma, in enhancing the fusion output in a primarily beam-injected twocomponent plasma, or in the production by if alone of an energetictail two-component plasma. Having determined the expected resonant-ion velocity distribution for different levels of $\mathrm{rf}$ heating, we are in a position to calculate the rf power cost for a certain rate of fusion reactions.

The $D-T$ nuclear power produced per unit volume is

$$
P_{n}=4 \pi n_{T} W_{n} \int_{0}^{\infty} v^{2} d v \dot{f}(v) \sigma(v) v
$$

where $n_{T}$ is the tritium density, $w_{n}$ the nuclear energy released per $D-T$ fusion reaction, and $\sigma(v)$ the $D-T$ cross-section as a function of the deuteron velocity. The rf power per unit volume is proportional to wave-induced dispersion, $\xi$. Taking this factor of proportionality from (34), we find the ratio, $Q_{R F}$, of nuclear power produced to rf power input

$$
Q_{K F^{\prime}}=\frac{w_{n} \varangle v>n_{T} m_{D}(2 k T e)^{1 / 2}}{8 \pi^{1 / 2} \xi m_{e}^{1 / 2} n_{e} e^{4} \ln \Lambda}
$$


The $\sigma \mathrm{v}$ average is to be taken over the distribution of resonant ions (deuterons). Especially convenient in computer calculation here is Duane's fit [18] to the D-T fusion cross-section. For the conditions pertaining to the example of section 7, that is, $\mathrm{kT}=4 \mathrm{keV}$, using $\mathrm{Z}_{\text {eff }}=3$ in computing $\mathrm{R}_{j}$, Eq. (34), and using $W_{n}=22.4 \mathrm{MeV}$, the computed $Q_{R F}$ values are 0.20 for $\xi=100$, 0.38 for $\xi=30$, and 0.045 for $\xi=10$. The maximum value for $\langle\sigma v\rangle / \xi$, for the same conditions, occurs for $\xi=42$, at which value $Q_{R F}=0.45$.

For comparison, we consider the injection into the same tritium plasma of $I$ deuterons per $\mathrm{cm}^{3}$ per second with energy $\mathrm{E}_{\text {. }}$ The deuteron velocity distribution, in accordance with Eq. (40), is $f(v)=I / 4 \pi \alpha v^{2}$ for $m v^{2} / 2<E_{O^{\prime}} f(v)=0$ for $m v^{2} / 2>E_{0}$. Putting this distribution into (41) determines the nuclear power, while the injected power is simply $I E_{0}$. The ratio, $Q_{I}$, for nuclear power to injected power is then

$$
\mathrm{Q}_{\mathrm{I}}=\frac{\mathrm{n}_{\mathrm{T}} \mathrm{W}_{\mathrm{n}}}{\mathrm{E}_{\mathrm{O}}} \int_{\mathrm{V}_{\mathrm{O}}}^{0} \frac{\sigma \mathrm{v}}{\alpha} \mathrm{dv}, \frac{\mathrm{mv}_{\mathrm{O}}^{2}}{2} \equiv \mathrm{E}_{\mathrm{O}} .
$$

With $z_{\text {eff }}=3$ in computing $v_{\alpha}^{2}$, Eq. (3l), and with $k T=4 \mathrm{keV}$, $Q_{I}=0.42$ for $E_{O}=100 \mathrm{keV}, 0.74$ for $E_{0}^{\prime}=150 \mathrm{keV}$, and 0.81 for $\mathrm{E}_{\mathrm{O}}=200 \mathrm{keV}$. Comparing these two sets of $\mathrm{Q}$ values we see that the efficiency for fusion power production from a $\mathrm{Z}_{\text {eff }}=3$ twocomponent plasma produced by selective rf absorption comes within. a factor of two with the corresponding efficiency for a neutralinjection system.

For $Z_{\text {eff }}=1$, computation shows $Q_{R F}$ values significantly greater than unity. Figure 6 is a plot of $Q_{R F}$ versus rf power, 
parameterized by $\xi$, for various electron temperatures, with $\mathrm{z}_{\text {eff }}=1$ and temperature $\mathrm{T}_{j}=4 \mathrm{keV}$ for the background triton ions. The $Q_{R F}$ values decrease for very high rf powers and for very large electron temperatures because too large a fraction of the deuteron distribution then appears in the far tail (E>200 keV) past the maximum of the fusion cross-section.

Figure 7 illustrates the effect of changing $z_{\text {eff }}$ and also the effect of lowering the background ion temperature.

\section{SUMMARY}

We have examined in considerable detail the use of the fast hydromagnetic wave for heating large magnetically-confined toroidal plasmas. Landau and transit-time damping, which are coherent processes, can couple power effectively into the electrons while the ions can be rf-heated with high efficiency at the second harmonic of their cyclotron frequency or at the fundamental cyclotron frequency of a minority ion species. In a particular application of the latter principle, selective rf absorption by deuterons in a predominantly triton plasma can lead to a twocomponent velocity aistribution suitable for the production of abundant fusion reactions. The process is analyzed by a FokkerPlanck equation with a Kennel-Engelmann quasilinear-type rf diffusion term, and an explicit analytic solution is found for the one-dimensional case. Using the resonant-ion distribution function thus derived, and the Duane-formula fit to the D-T fusion cross-section, the computed efficiencies for the rf two-component formation process in a $Z=1$ plasma show $Q_{R F} \equiv$ (nuclear power out)/(rf power in) values significantly above unity. 


\section{ACKNOWLEDGMENTS}

It is a pleasure to acknowledge a number of helpful discussions with Dr. W. M. Hooke, Dr. D. Jassby, and Dr. F. W. Perkins. This work was supported by the U.S. Atomic Energy Commission Contract $\operatorname{AT}(11-1)-3073$ 


\section{REFERENCES}

[1] DAWSON, J. M., FURTH, H. P., and TENNEY, F. H., PhYS . Rev. Letters 26 (1971) 1156 .

[2] STIX, T. H., Phys. Fluids $\underline{1}$ (1958) 308 .

[3] YOSHIKAWA, S., ROTHMAN, M. A., and SINCLAIR, R. M., PhYs. Rev. Letters 14 (1965) 214 .

[4] ADAM, J., and SAMAIN, A., Fontenay-aux-Roses, Report EURCEA $-F C-579$ (1971) 29 .

[5] PERKINS, F. W., Symposium on Plasma Heating and Injection, Varenna (1972) 20 .

[6] JASSBY, D. L., 2nd Conference on RF Heating, Lubbock, Texas, (1974).

[.7] HOOKE, W. M., ROTHMAN, M. A., AVIVI, P., ADAM, J., PhYs. Fluids $\underline{5}$ (1962) 864 .

[8] CHUNG, K., and ROTHMAN, M. A., Phys. Fluids 10 (1967) 2634.

[9] ADAM, J., CHANCE, M., EUBANK, H., GETTY, W., HINNOV, E., HOOKE, W. M., HOSEA, J., JOBES, F., PERKINS, F., SINCLAIR, R., SPERLING, J., and TAKAHASHI, H., IAEA-CN-33/A3-2 (1974).

[10] STIX, T. H., The Theory of Plasma Waves, McGraw-Hill, New York (1962), Eq. (5-26).

[11] BERNSTEIN, I. B., and TREHAN, S: K., Nuclear Fusion 1 (1960) 3, Eq. (24).

[12] CHANCE, M., and PERKINS, F. W., private communication. See also Ref. [9], Figure 6.

[13] STIX, T. H., (1962), op. cit., Eq. (8-10).

[14] ADAM, J., and SAMAIN, A., op. cit. An incorrect expression is used for the circumference of a circle in Eq. (2), p. 32, and the value cited there for $\overline{\mathrm{P}}$ is too large by a factor of 2 . 
[15] KENNEL, C. F., and ENGELMANN, F., Phys. Fluids $\underline{9}$ (1966) 2377 .

[16] SPITZER, L., Jr., The Physics of Fully Ionized Gases, 2nd Revised Edition, Interscience, New York (1962).

[17] COHEN, R. S., SPITZER, L., Jr., and ROUTLY, P. M., PhYs. Rev. 80 (1950) 230 .

[18] DUANE, B. H., Battelle Pacific Northwest Laboratories, BNWL-1685 (1972) 75 . 


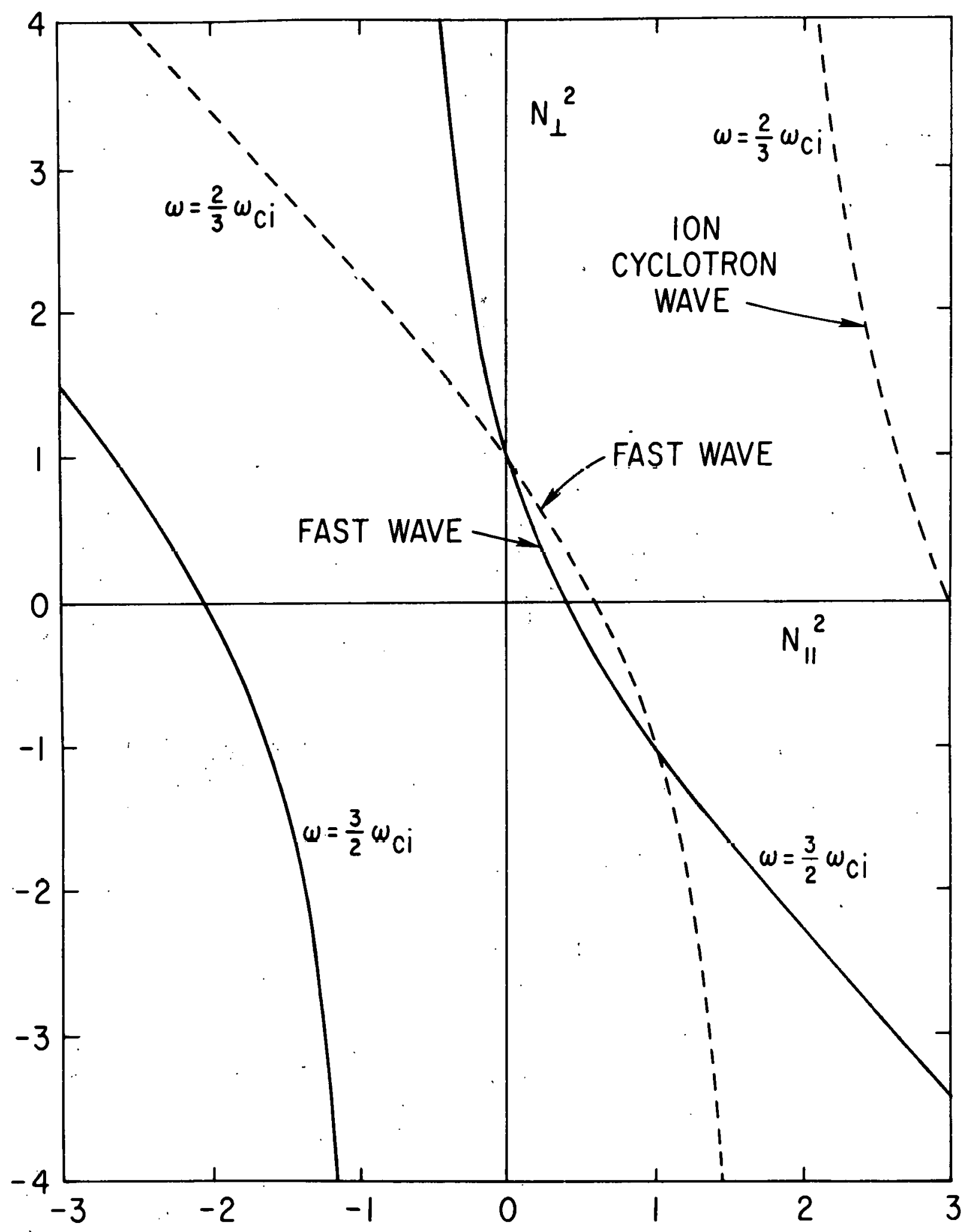

753034

Fig. 1. Dispersion curves for hydromagnetic waves above and below the ion cyclotron frequency. $N_{n}$ and $N_{\perp}$ are the Alfién refractive indices. 


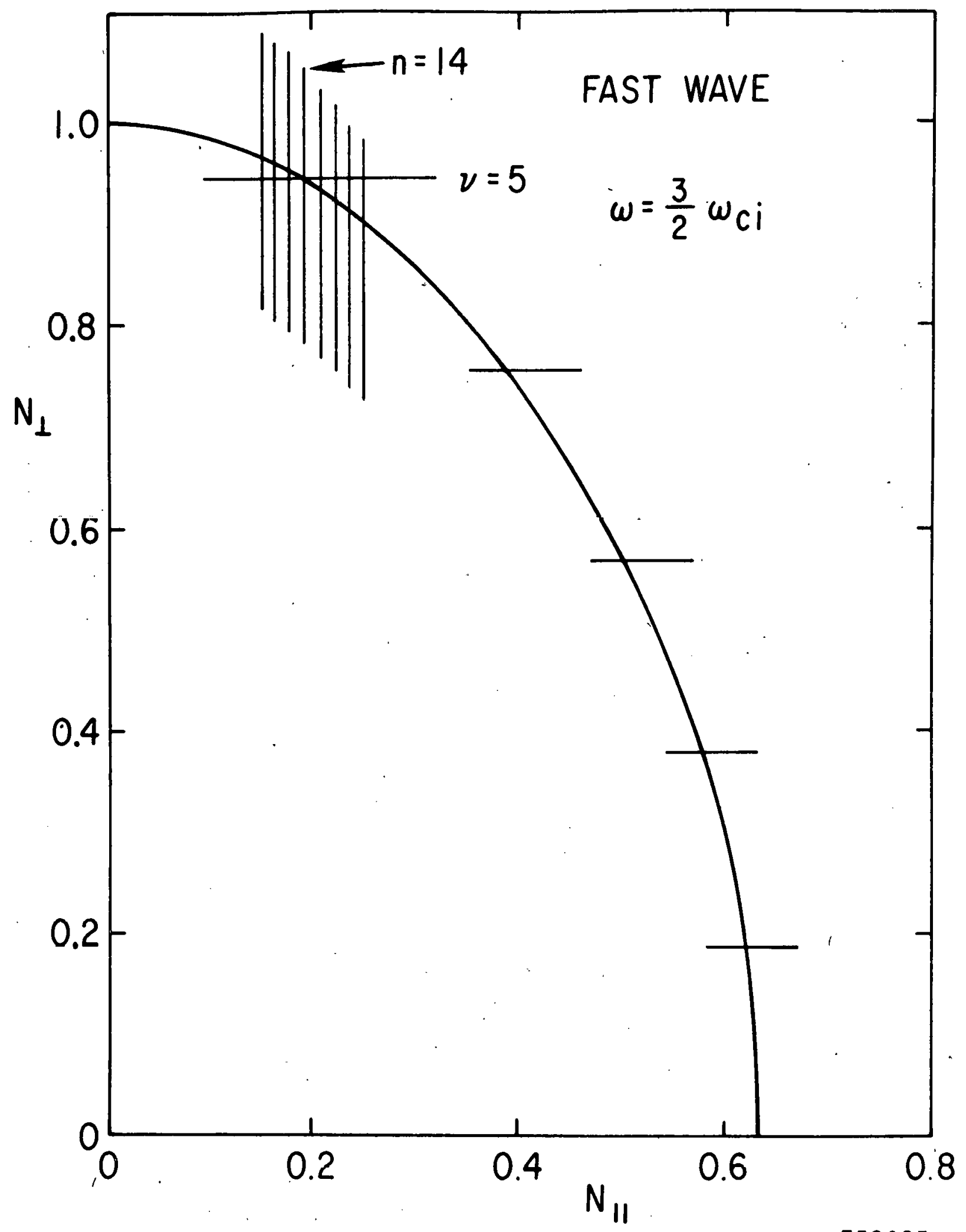

753035

Fig. 2. Dispersion curve for the hydromagnetic fast wave at $\omega=1.5 \omega \mathrm{w}^{\circ}$. The curve here retraces the solid curve through just the firstiquadrant of Fig. 1. The grid lines correspond to the different poloidal and toroidal mode numbers. Actually, each poloidal $(\nu)$ mode is a $(2 \nu-1)$-fold multiplet, and each toroidal (n) mode is a doublet. 


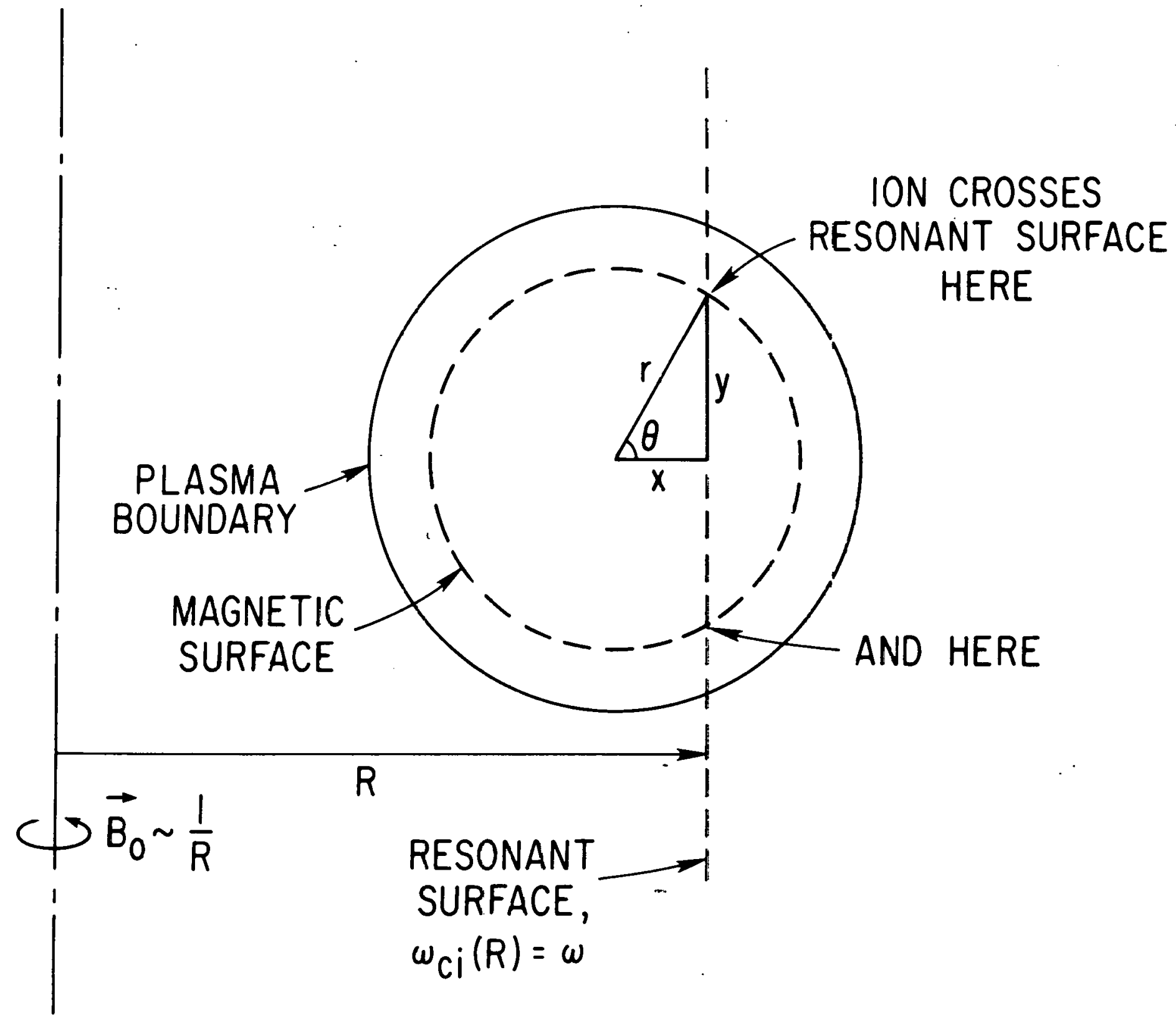

Fig. 3. Geometry for cyclotron resonance excitation in a torus. Due to the $1 / \mathrm{R}$ dependence of $\mathrm{B}$, the resonance region is a vertical cylindrical shell. Ions moving along a magnetic line of force and confined to a magnetic surface will intersect the resonance shell above and below the midplane. 


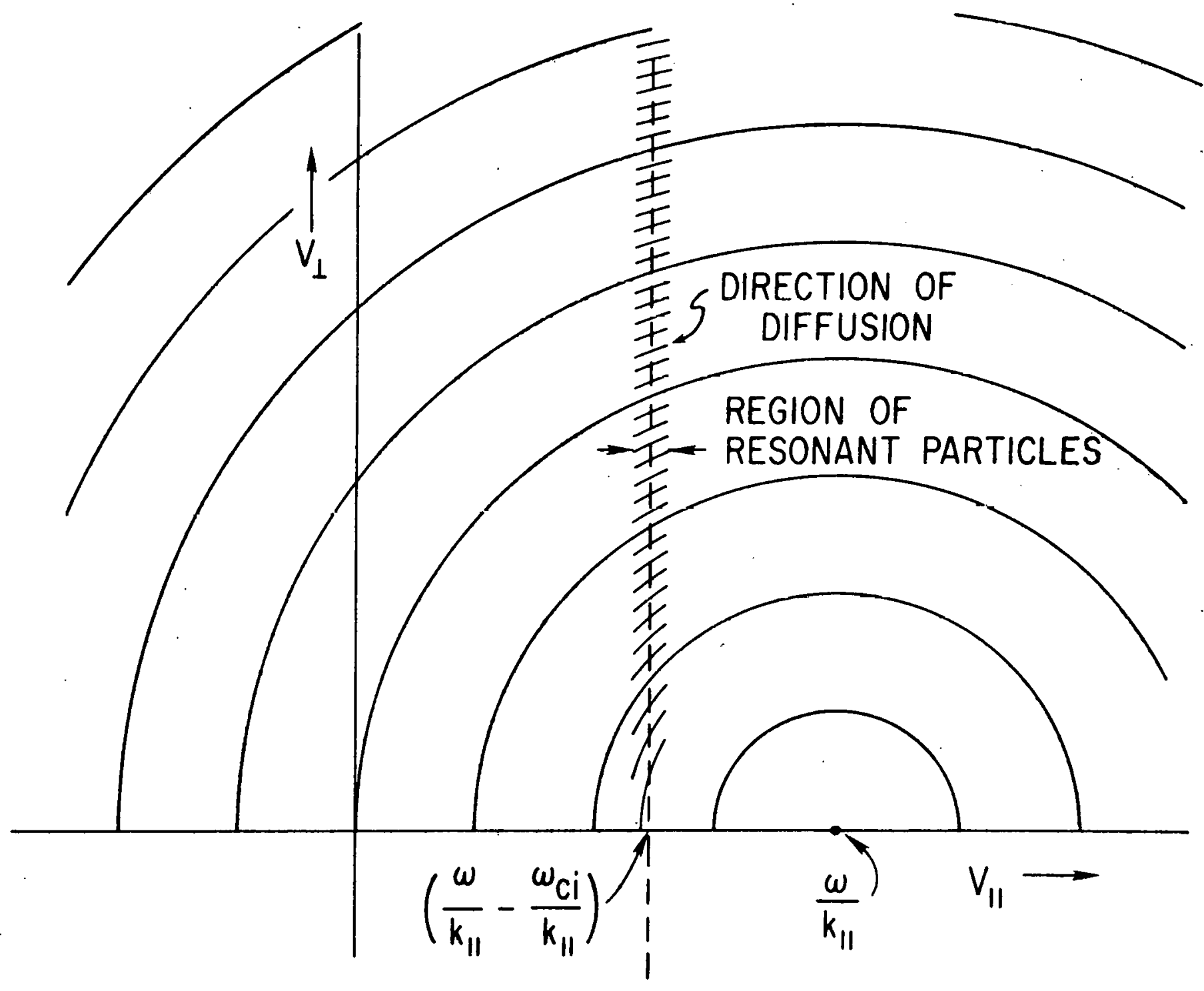

753038

Fig. 4. Contours for quasilinear diffusion. The quasilinear diffusion operator is a gradient operator in velocity space along the indicated circles. Diffusion takes place just in the circumferential direction, but only where the resonance condition is satisfied, i.e., only where the circles intersect the vertical lines. 


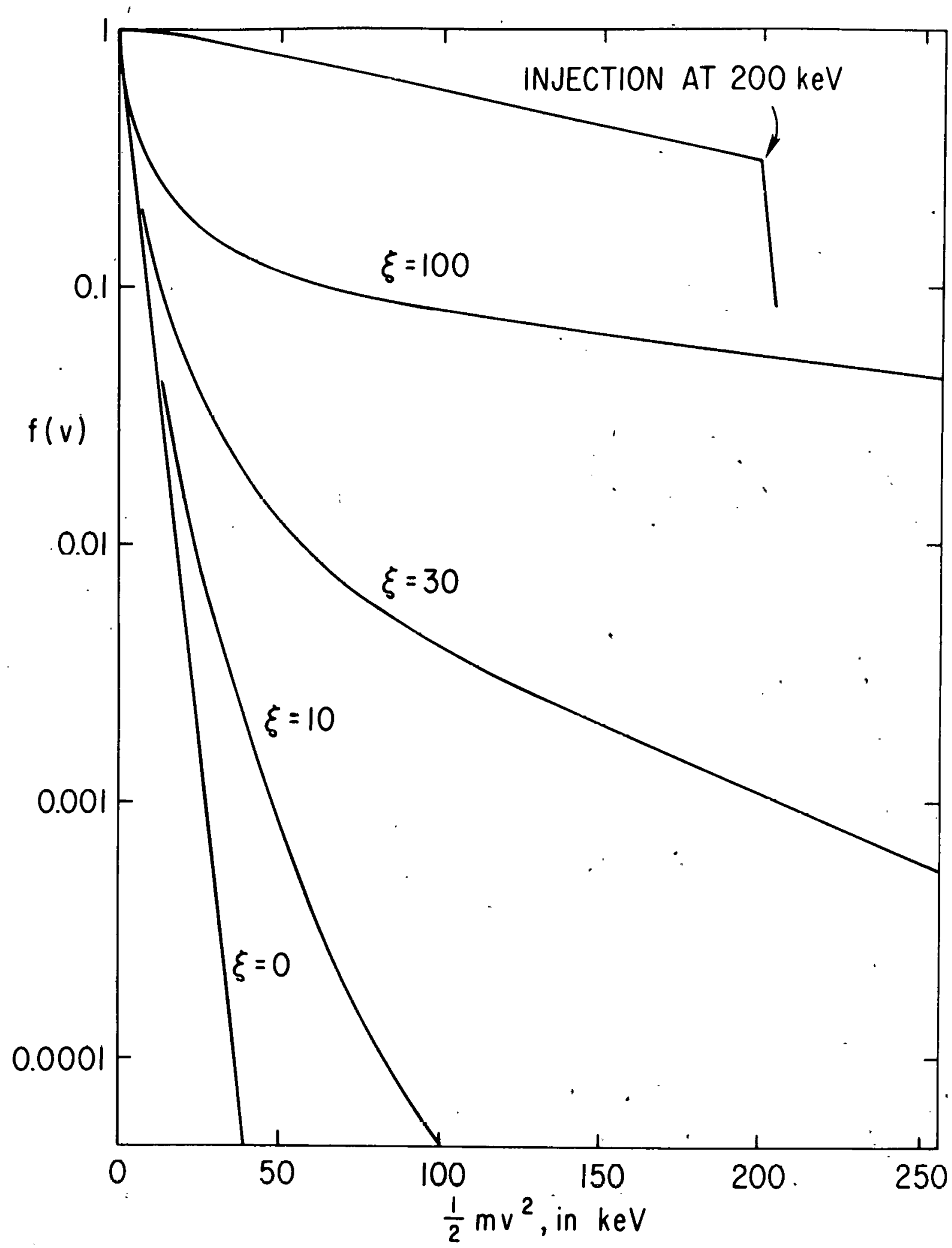

753032

Fig. 5. Plots of $f(v)$ versus $E$ for the plasma parameters of the example in Section 7, and for different levels ( $\xi$ ) of $r f$ excitation at the minority-species cyclotron frequency. $z_{\text {ff }}=3$. The ion velocity distribution for injection at $200 \mathrm{keV}$ int $\delta^{f}$ 解 same plasma is also shown. 


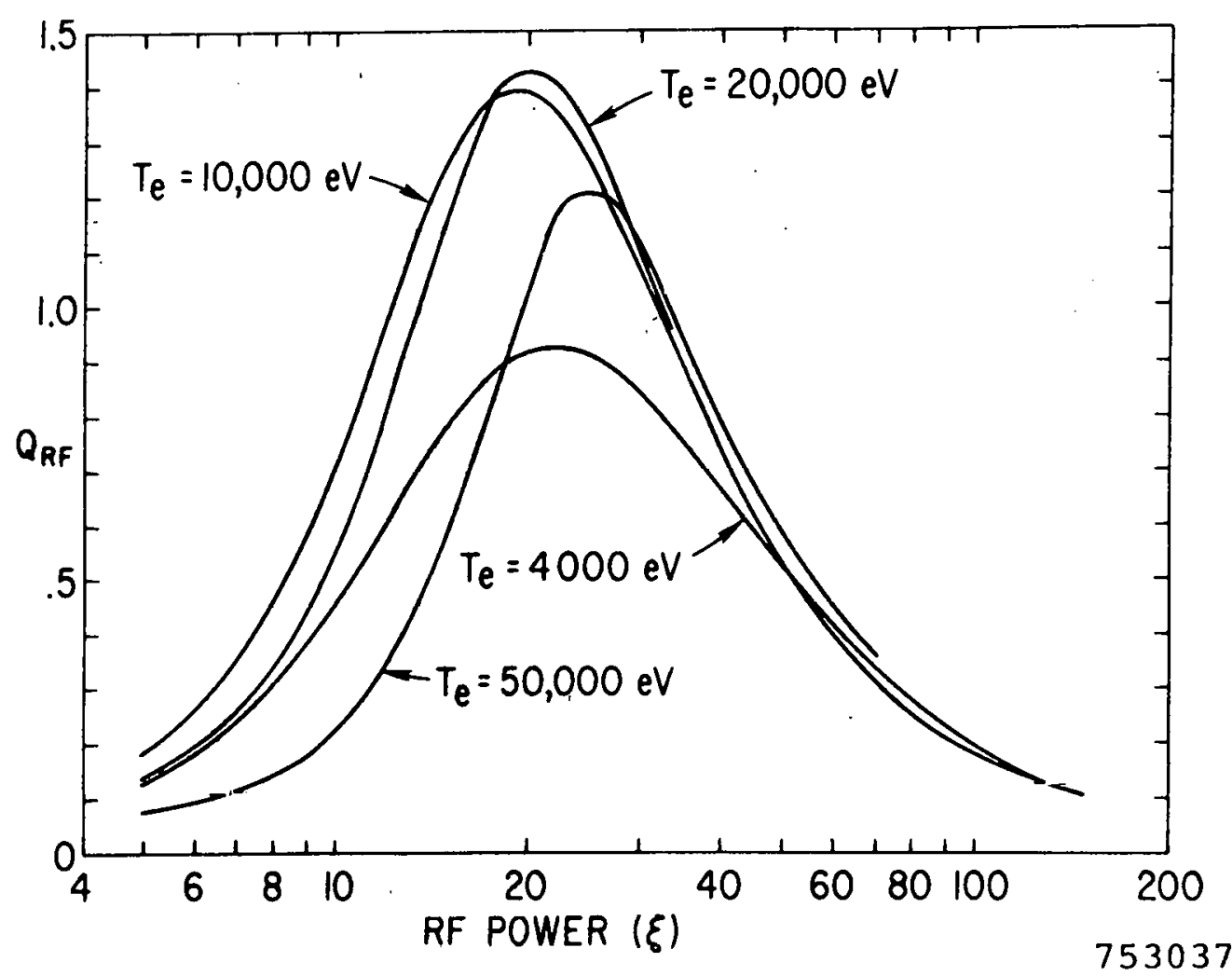

Fig. 6. $Q_{R F} \equiv$ (nuclear power out)/(rf power in is plotted versús rf power per unit volume selectively absorbed by the resonant minority deuterons. $z_{\text {eff }}=1$.

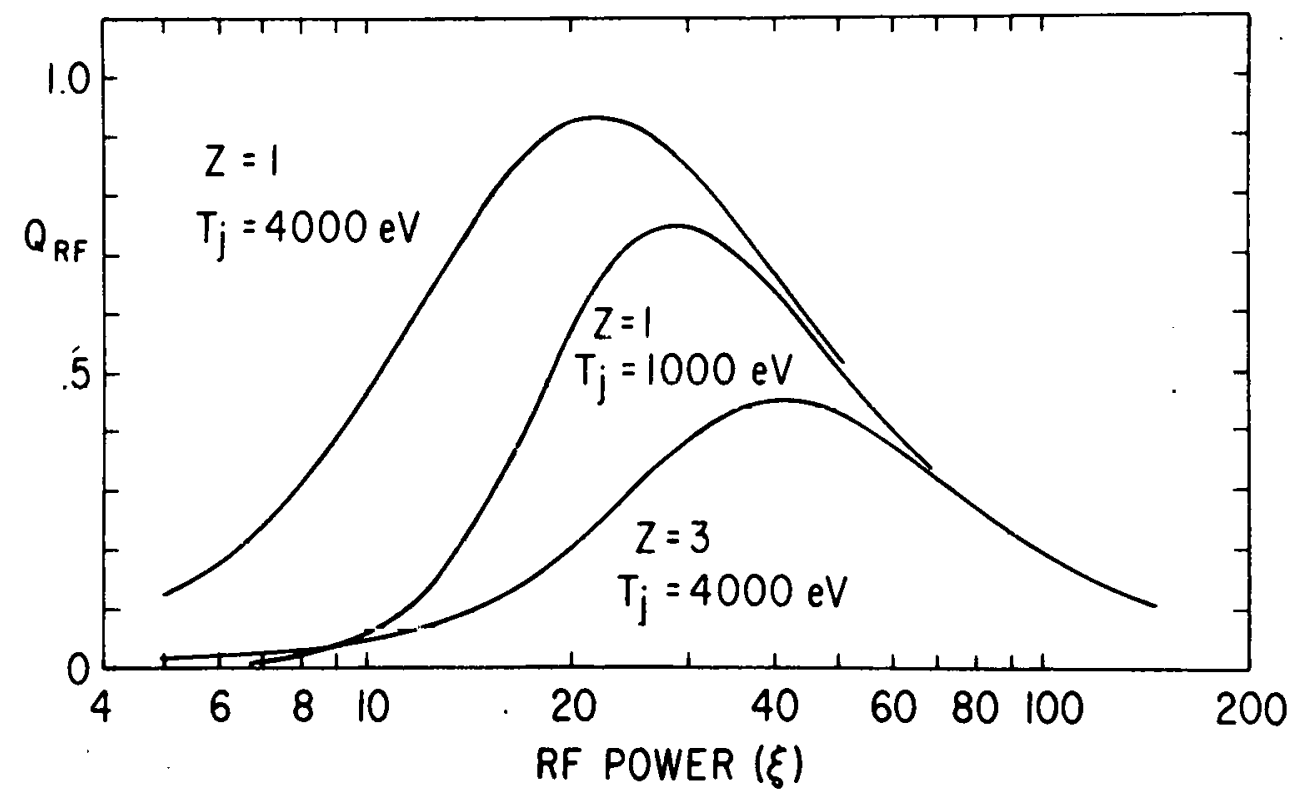

753036

Fig. 7. $Q_{\mathrm{RF}}$ versus $\mathrm{rf}$ power for $\mathrm{kT}=\mathrm{keV}$, for $z$ ff $=1$ and 3 , and for temperatures of $1^{e} \mathrm{keV}$ and $4 \mathrm{keV}$ for the background triton ions. 


\section{NOTICE}

This report was prepared as an account of work sponsored by the United States Government. Neither the United States nor the United States Atomic Energy Commission, nor any of their employees, nor any of their contractors, subcontractors, or their employees, makes any warranty, express or implied, or assumes any legal liability or responsibility for the accuracy, completeness or usefulness of any information, apparatus, product or process disclosed, or represents that its use would not infringe privately owned rights. 